\title{
VOLTERRA INTEGRAL EQUATIONS IN BANACH SPACE
}

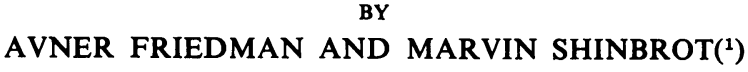

Chapter 1. Existence, Uniqueness, and Differentiability

1. Statement of results on existence and uniqueness, p. 133

2. Preliminaries, p. 135

3. Proof of Theorem 1, p. 137

4. Proofs of Theorems 2, 4, p. 143

5. Further properties of $W$, p. 145

6. Global bounds on $W$, p. 147

7. Differentiability of solutions, p. 149

8. Other types of $A(t)$, p. 150

9. The case $h(0)=0$, p. 153

10. Applications and generalizations, p. 154

11. Some results in Hilbert space, p. 155

Chapter 2. Global Bounds and Asymptotic Behavior

1. Preliminaries, p. 157

2. $S$ in $L^{p}(0, \infty ; B(X))$ for $2 \leqq p<\infty$, p. 159

3. $S$ in $L^{1}(0, \infty ; B(X))$, p. 164

4. Further bounds on $S$, p. 166

5. Further bounds on $S$ (continued), p. 169

6. Representation of solutions, p. 171

7. A uniqueness theorem and its applications, p. 173

8. The resolvent $R_{\delta}$, p. 175

9. Asymptotic behavior, p. 177

Introduction. In Chapter 1 we consider the equation

$$
u(t)=k(t)-\int_{0}^{t} h(t-\tau) A(\tau) u(\tau) d \tau
$$

where $u, k$ are functions with values in a Banach space $X, h$ is a scalar function, and $A(t)$ is an unbounded operator in $X$ which generates an analytic semigroup. It is assumed that $A(t) A^{-1}(0)$ is Hölder continuous and that $k, h$ have certain differentiability properties.

Received by the editors March 2, 1966.

(1) The first author was supported by NASA Grant NGR 14-007-021. The second author was supported by National Science Foundation Grant GP-4586. 
In case $h(t) \equiv 1,(1)$ is equivalent to

$$
\begin{array}{r}
d u / d t+A(t) u=f(t), \\
u(0)=k(0)
\end{array}
$$

where $f(t)=\dot{k}(t)$. The system (2), (3) has been thoroughly studied in the literature (see [9], [10], [15]-[18]). Existence, uniqueness, and differentiability theorems are well known. The solution can be represented in terms of the fundamental solution. Local and global bounds on the fundamental solutions are also known. Most of these results are stated in $\$ 1.2$ below.

We prove, in Chapter 1, that essentially all these results are valid also for the more general case of equation (1), provided $h(0)>0$. Our treatment is based on the following scheme. We reduce (1) to an equation of the form $u=T(u)+F(u)$, where $F(u)$ is "small"; in case $h(t) \equiv 1$ the equation reduces to $u=T(u)$. We then treat the reduced problem as a perturbation problem, employing simple fixed-point theorem techniques.

The treatment of the fundamental solution $W$ presents a special difficulty since $A W$ is generally nonintegrable (already for $h \equiv 1$ ). Thus what might be considered the natural integral equation for $W$ (see (1.1.5)) does not make any sense. One way to handle this difficulty is to consider not $W$ but rather $W x$ for $x$ in the domain of some $A^{\mu}(\mu>0)$. In this way one obtains a fundamental solution which may, however, be an unbounded operator. Another method (given in \$1.5) involves replacing (1.1.5) by a "weaker" equation whose solution is a bounded and smooth operator function and showing that this solution is the fundamental solution. This second method requires additional assumptions on $A(t)$; in case $A(t)$ is an elliptic differential operator, these assumptions roughly amount to assuming that the leading coefficients are independent of $t$.

In Chapter 2, we consider the case where $A(t)$ is independent of $t$. We then employ Laplace transforms to obtain very precise global bounds on the fundamental solution. Under certain assumptions, we prove that the fundamental solution belongs to $L^{p}(0, \infty ; B(X))$ for all $1 \leqq p \leqq \infty$. This result is sharp, for all but a small part of the main condition is necessary as well as sufficient in all cases and the entire condition is necessary and sufficient in the important case when $h(t)$ is monotone decreasing and nonnegative.

The global bounds proved in Chapter 2 are used to derive results on the asymptotic behavior of solutions of (1). In fact, our original motivation for deriving the above mentioned bounds on the fundamental solution was to study the asymptotic behavior of solutions of (1) in a fashion similar to [5], where the case where $u(t)$, $k(t), A(t)$ are all scalar functions was treated. 


\section{Chapter 1. Existence, Uniqueness, and Differentiability}

1. Statement of results on existence and uniqueness. Let $X$ be a complex Banach space, and let $A(t)$ be closed, densely defined, linear operators with domain $D(A)$ independent of $t$. We need the following assumptions:

(A) The resolvent $(\lambda I-A(t))^{-1}$ exists for all $0 \leqq t<T, \operatorname{Re} \lambda \leqq 0$, and

$$
\left\|(\lambda I-A(t))^{-1}\right\| \leqq \frac{C}{1+|\lambda|}
$$

where $C$ is a constant independent of $t, \lambda$.

(B) For any $t, \tau$ in $[0, T)$,

$$
\left\|(A(t)-A(\tau)) A^{-1}(\tau)\right\| \leqq C^{\prime}|t-\tau|^{\alpha}
$$

where $C^{\prime}, \alpha$ are positive constants (independent of $t, \tau$ ), and $\alpha \leqq 1$.

Note that (1.2) implies that $A(t) A^{-1}(\tau)$ is a bounded operator, its norm is bounded by a constant independent of $t, \tau$, and

$$
\left\|(A(t)-A(\tau)) A^{-1}(s)\right\| \leqq C^{\prime \prime}|t-\tau|^{\alpha}
$$

for any $t, \tau, s$ in $[0, T)$.

Under the assumption (A), the fractional power $A^{\rho}(t)$ of $A(t)$ is defined for any real $\rho$ (see, for instance, [15]). For $\rho<0, A^{\rho}(t)$ is a bounded operator, and, for $\rho>0, A^{\rho}(t)=\left(A^{-\rho}(t)\right)^{-1}$. Thus, as the inverse of a bounded operator, $A^{\rho}(t)$ is a closed (but generally unbounded) operator; its domain contains $D(A)$ if $\rho<1$.

We shall consider integral equations of the form

$$
u(t)=k(t)-\int_{0}^{t} h(t-\tau) A(\tau) u(\tau) d \tau \quad(0 \leqq t<T)
$$

where $h$ is a given scalar function, $k(t)$ is a given function from $[0, T)$ into $X$, and the solution $u(t)$ is a function from $[0, T)$ into $D(A)$. We need the following conditions on $h, k$ :

(H) $h(0)>0, h \in C^{1}[0, T), h(t)$ is absolutely continuous in $[0, T)$ and $\vec{h} \in L^{p}(0, T)$ for some $p>1$.

(K) $k(0) \in D\left(A^{\mu}(0)\right)$ for some $0<\mu \leqq 1, \dot{k}(t)=\lim _{h \rightarrow 0}[k(t+h)-k(t)] / h$ exists in $X$ for all $0 \leqq t<T$, and is uniformly Hölder continuous, i.e.,

$$
\|\dot{k}(t)-\dot{k}(s)\| \leqq c|t-s|^{\beta}
$$

for all $t, s$ in $[0, T)$, where $c, \beta$ are positive constants, and $\beta \leqq 1$.

Note that $h(t)$ is uniformly Hölder continuous in $(0, T)$ with exponent $1 / q$, where $1 / p+1 / q=1$.

Definition. A function $u(t)$ from $[0, T)$ into $D(A)$ is called a solution of (1.4) if $u(t)$ is continuous in $[0, T)$, if $A(t) u(t)$ is continuous in $(0, T),\|A(t) u(t)\|$ is integrable in $[0, T-\varepsilon)$ for any $\varepsilon>0$, and, finally, if (1.4) is satisfied. If it is also true that $t^{\delta}\|A(t) u(t)\|$ remains bounded for some $0 \leqq \delta<1$ as $t \rightarrow 0$, then we say that $u$ is a strong solution. 
THEOREM 1. Assume that (A), (B), (H), (K) hold. Then there exists a unique strong solution $u(t)$ of (1.4). $t^{1-\mu} A(t) u(t)$ remains bounded as $t \rightarrow 0, u(t)$ is uniformly Hölder continuous with exponent $\mu$ in $[0, T)$ and, moreover, if $k(0) \in D(A)$ then $A(t) u(t)$ and $d u(t) / d t$ are continuous in $[0, T)$.

COROLlaRY. If the assumption $(\mathrm{H})$ is strengthened by requiring $\ddot{h}(t)$ to be Hölder continuous in some interval $[0, \varepsilon)$ with $\varepsilon>0$, then there cannot exist more than one solution of (1.4).

We are interested not only in proving the existence of solutions, but also in representing them in terms of a "fundamental solution," i.e., in terms of an operator function $W(t, s)$ satisfying

$$
W(t, s)=I-\int_{s}^{t} h(t-\tau) A(\tau) W(\tau, s) d \tau \quad(0 \leqq s \leqq t<T) .
$$

However, $\|A(\tau) W(\tau, s)\|$ is not integrable in $\tau$ even in the case $h(t) \equiv 1$ (in fact, its growth at $\tau=s$ may be like $\left.(\tau-s)^{-1}\right)$; thus the integral in (1.5) cannot be taken in the same natural sense as (1.4).

Instead of considering (1.5) directly, we first consider the equation

$$
W_{\mu}(t, s)=A^{-\mu}(s)-\int_{s}^{t} h(t-\tau) A(\tau) W_{\mu}(\tau, s) d \tau \quad(0 \leqq s \leqq t<T)
$$

for any $0<\mu \leqq 1$.

Definition. An operator-valued function $W_{\mu}(t, s)$ defined for $0 \leqq s \leqq t<T$ is said to be a solution of (1.6) if it is continuous in $(t, s)$ (in the strong topology) for $0 \leqq s \leqq t<T$, if $A(t) W_{\mu}(t, s)$ is continuous in $t$ (in the strong topology) for $0 \leqq s<t<T$, if $\left\|A(t) W_{\mu}(t, s)\right\|$ is integrable in $t$ in any interval $(s, T-\varepsilon)$ with $\varepsilon>0$ and, finally, if (1.6) is satisfied. If also, for some $0 \leqq \delta<1,(t-s)^{\delta}\left\|A(t) W_{\mu}(t, s)\right\|$ remains bounded as $t-s \rightarrow 0$, then it is called a strong solution.

THEOREM 2. Assume that (A), (B), (H) hold. Then there exists a unique strong solution of (1.6). Furthermore, for $0 \leqq s<t<T$,

$$
\left\|A^{\rho}(t) W_{\mu}(t, s)\right\| \leqq \operatorname{const}(t-s)^{\mu-\rho} \quad(\mu \leqq \rho<1+\alpha) .
$$

An analogue of the Corollary to Theorem 1 is also valid.

Definition. The operator function $W(t, s)=W_{u}(t, s) A^{\mu}(s)$ is called the fundamental solution of (1.4) or (1.5).

To justify this definition we shall prove the following theorem.

TheOrem 3. Assume that (A), (B), (H) hold. Then $W(t, s)$ is independent of $\mu$, i.e., if $x \in D\left(A^{\mu}\right) \cap D\left(A^{v}\right)$ then $W_{\mu}(t, s) A^{\mu}(s) x=W_{v}(t, s) A^{v}(s) x$. Furthermore, for any element $x$ which belongs to some $D\left(A^{\mu}(s)\right)$, the function $W(t, s) x$ satisfies the equation

$$
W(t, s) x=x-\int_{s}^{t} h(t-\tau) A(\tau) W(\tau, s) x d \tau,
$$

and $\|A(t) W(t, s) x\| \leqq$ const $(t-s)^{\mu-1}$.

Thus (1.5) is satisfied in the weaker sense of (1.8). 
Proof. Set $w(t)=W_{\mu}(t, s) A^{\mu}(s) x \equiv W(t, s) x$. Applying both sides of (1.6) to $A^{\mu}(s) x$, we find that $w(t)$ is a solution of

$$
w(t)=x-\int_{s}^{t} h(t-\tau) A(\tau) w(\tau) d \tau
$$

and, by (1.7), $\|A(t) w(t)\| \leqq$ const $(t-s)^{\mu-1}$. Thus (1.8) and the inequality following it are satisfied. Since $w(t)$ is also a strong solution, it is unique (by Theorem 1). Hence, if $x$ also belongs to $D\left(A^{\nu}(s)\right)$ with some $0<\nu \leqq 1$ then

$$
W_{\mu}(t, s) A^{\mu}(s) x=W_{\nu}(t, s) A^{\nu}(s) x,
$$

i.e., $W(t, s)$ is independent of $\mu$.

In $\S 1.5$ we shall obtain further results on $W$.

We now state a representation theorem.

THEOREM 4. Assume that (A), (B), (H), (K) hold, that $\dot{k}(t) \in D\left(A^{\mu}(t)\right)$, and that $A^{\mu}(t) \dot{k}(t)$ is continuous for $t \in[0, T)$. Then the solution $u(t)$ of $(1.4)$ (whose existence is asserted in Theorem 1) can be represented in the form

$$
u(t)=W(t, 0) k(0)+\int_{0}^{t} W(t, s) \dot{k}(s) d s .
$$

The proofs of Theorem 1 and its corollary are given in $\$ 3$, and the proofs of Theorems 2,4 are given in $\$ 4$. In $\$ 2$ we collect several results which will be needed throughout this chapter.

2. Preliminaries. In this section we assume that the $A(t)$ are as in Theorems 1-3. Then, as proved by Sobolevskiir [15] and Tanabe [16], [17], [18], there exists an operator-valued function $U(t, \tau)$ defined for $0 \leqq \tau \leqq t<T$ and having the following properties:

For fixed $\tau \in[0, T), U(t, \tau)$ and $\partial U(t, \tau) / \partial t$ are continuous in $t$ (in the strong topology) in the intervals $[\tau, T)$ and $(\tau, T)$, respectively, the range of $U(t, \tau)$ is in the domain $D(A)$ of $A(t)$, and

$$
\begin{aligned}
\partial U(t, \tau) / \partial t+A(t) U(t, \tau) & =0 \quad(\tau<t \leqq T), \\
U(\tau, \tau) & =I ;
\end{aligned}
$$

the derivative $\partial U / \partial t$ is taken in the strong topology. $U(t, \tau)$ is also continuous (in the strong topology) in $(t, \tau)$ for $0 \leqq \tau \leqq t<T$.

We mention further results which they have proved. If $f(t)$ is any bounded function from $[0, T)$ into $X$ which is uniformly Hölder continuous in any interval $(\varepsilon, T-\varepsilon)$ with $\varepsilon>0$, and if $u_{0}$ is any element in $X$, then the system

$$
\begin{aligned}
d u / d t+A(t) u & =f(t) \quad(0<t<T), \\
u(0) & =u_{0}
\end{aligned}
$$


has a unique solution, continuous in $[0, T)$ and continuously differentiable in $(0, T)$. The solution can be represented in the form

$$
u(t)=U(t, 0) u_{0}+\int_{0}^{t} U(t, s) f(s) d s .
$$

If $u_{0} \in D(A)$ and $f(t)$ is uniformly Hölder continuous in any interval $[0, T-\varepsilon)$ with $\varepsilon>0$, then $d u(t) / d t$ and $A(t) u(t)$ are continuous in $[0, T)$.

Analogous results hold in case $f(t)$ is an operator-valued function. In particular, $A(t) \int_{0}^{t} U(t, s) d s$ is continuous (in the strong topology) for $0 \leqq t<T$. If we use this result with $t=0$ replaced by $t=T_{0}$, for any $0 \leqq T_{0}<T$, we get:

$$
\left\|A(t) \int_{T_{0}}^{t} U(t, s) d s\right\| \leqq \text { const }<\infty \quad\left(T_{0}<t<T\right)
$$

where the constant is independent of $T_{0}$.

$U(t, \tau)$ is called the fundamental solution of the equation $d v / d t+A(t) v=0$.

The following inequalities are valid:

$$
\begin{aligned}
&\left\|A^{\rho}(t) U(t, \tau) A^{-\sigma}(\tau)\right\| \leqq C(t-\tau)^{\sigma-\rho}(0 \leqq \sigma \leqq \rho<1+\alpha), \\
&\left\|A^{\rho}(0)[U(t+\Delta t, \tau)-U(t, \tau)] A^{-\sigma}(\tau)\right\| \leqq C(\Delta t)^{\nu-\rho}(t-\tau)^{\sigma-\nu}, \\
&(0 \leqq \rho \leqq 1,0 \leqq \sigma \leqq \nu<1+\alpha, 0 \leqq \nu-\rho \leqq 1),
\end{aligned}
$$

where $\Delta t>0, C$ is a constant, and $\alpha$ is the constant appearing in (1.2).

In $\S \S 3,4$ we shall need the following lemma.

LEMMA 1. Let $0 \leqq T_{0}<T$ and let $f(t)$ be a continuous function from $\left[T_{0}, T\right)$ into $X$, satisfying:

$$
\|f(t)\| \leqq \Omega v(t), \quad\|f(t)-f(s)\| \leqq \Omega \nu(t)(t-s)^{\mu} \quad\left(T_{0} \leqq s \leqq t<T\right)
$$

where $\nu(t)$ is a positive and monotone increasing function, $\Omega, \mu$ are positive constants and $\mu \leqq 1$. Then the function

$$
F(t)=\int_{T_{0}}^{t} U(t, s) f(s) d s \quad\left(T_{0} \leqq t<T\right)
$$

satisfies:

$$
\|A(t) F(t)\| \leqq C \Omega \nu(t)
$$

where $C$ is a constant independent of $T_{0}, f$.

Proof. Write

$$
\begin{aligned}
A(t) F(t) & =\left\{A(t) \int_{T_{0}}^{t} U(t, s) d s\right\} f(t)+A(t) \int_{T_{0}}^{t} U(t, s)[f(s)-f(t)] d s \\
& \equiv J(t) f(t)+A(t) g(t)
\end{aligned}
$$

and set

$$
G_{\varepsilon}(t)=\int_{T_{0}}^{t-\varepsilon} A(t) U(t, s)[f(s)-f(t)] d s
$$


By (2.7) with $\rho=1, \sigma=0$, the integral of $G_{\varepsilon}(t)$ exists for all $\varepsilon \geqq 0\left(\varepsilon<t-T_{0}\right)$ and $G_{\varepsilon}(t) \rightarrow G_{0}(t)$ in $X$, as $\varepsilon \rightarrow 0$. Since also

$$
\int_{T_{0}}^{t-\varepsilon} U(t, s)[f(s)-f(t)] d s \rightarrow \int_{T_{0}}^{t} U(t, s)[f(s)-f(t)] d s \text { in } X,
$$

and since $A(t)$ is a closed operator, we conclude that $A(t) g(t)=\lim G_{\varepsilon}(t)$. Since $\left\|G_{\varepsilon}(t)\right\| \leqq$ const $\Omega \nu(t)$, the same bound holds for $\|A(t) g(t)\|$. Next, by (2.6), $\|J(t) f(t)\|$ is also bounded by const $\Omega \nu(t)$. By (2.12) we then see that (2.11) holds.

We conclude with a result on differentiability of solutions also due to Sobolevskii and Tanabe, and another on analyticity of solutions due to Sobolevskiir and Komatzu [12]:

Let $A(t) A^{-1}(0)$ have $m$ strongly continuous derivatives, let $f(t)$ have $m$ strong derivatives, and let $f^{(m)}(t)$ and $A^{(m)}(t) A^{-1}(0)$ be uniformly Hölder continuous in closed subsets of $(0, T)$. Then every solution of $(2.3)$ has $(m+1)$ strong derivatives in $(0, T)$ uniformly Hölder continuous in closed subsets of $(0, T)$.

If $A(t) A^{-1}(0)$ and $f(t)$ have strong derivatives of any order, and if

$$
\left\|A^{(m)}(t) A^{-1}(0)\right\| \leqq M_{0} M^{m} m !, \quad\left\|f^{(m)}(t)\right\| \leqq M_{0} M^{m} m !
$$

for all $t \in[0, T), m=1,2, \ldots$, where $M_{0}, M$ are constants, then every solution of (2.3) is analytic in $(0, T)$, i.e., in every closed subinterval of $(0, T)$,

$$
\left\|u^{(m)}(t)\right\| \leqq N_{0} N^{m} m !
$$

for some constants $N_{0}, N$ and for all positive integers $m$.

3. Proof of Theorem 1. We shall reduce the problem of solving (1.4) to a problem of solving a certain "integro-differential" equation. First we proceed formally. Differentiating (1.4) we get

$$
\frac{d u(t)}{d t}+h(0) A(t) u(t)=\dot{k}(t)-\int_{0}^{t} h(t-\tau) A(\tau) u(\tau) d \tau .
$$

Writing the last term in the form

$$
\int_{0}^{t} h(t-\tau)[A(t)-A(\tau)] u(\tau) d \tau-A(t) \int_{0}^{t} h(t-\tau) u(\tau) d \tau
$$

and introducing the function

$$
\tilde{u}(t)=u(t)+\frac{1}{h(0)} \int_{0}^{t} h(t-\tau) u(\tau) d \tau
$$

we reduce the equation (3.1) and the initial condition $u(0)=k(0)$ to

$$
\begin{aligned}
d \tilde{u} / d t+h(0) A(t) \tilde{u} & =\dot{k}(t)+f(\tilde{u} ; t), \\
\tilde{u}(0) & =k(0),
\end{aligned}
$$


where

$$
\begin{aligned}
f(\tilde{u} ; t)= & \int_{0}^{t} h(t-\tau)\left\{[A(t)-A(\tau)] A^{-1}(\tau)\right\} A(\tau) u(\tau) d \tau \\
& +\frac{h(0)}{h(0)} u(t)+\frac{1}{h(0)} \int_{0}^{t} \ddot{h}(t-\tau) u(\tau) d \tau .
\end{aligned}
$$

We observe that (3.2) is a classical linear Volterra integral equation, which can therefore be inverted. That is, whenever $t_{0}<T$ and whenever $\tilde{u}(t)$ is a continuous function from $\left[0, t_{0}\right]$ into $X$, there exists a unique continuous solution $u(t)$ of (3.2). Furthermore, $u(t)$ can be written in the form

$$
u(t)=\tilde{u}(t)+\int_{0}^{t} n(t-\tau) \tilde{u}(\tau) d \tau,
$$

where $n(t)$ is a function depending only on $h(t)$, having the same smoothness properties (continuity, Hölder continuity, absolute continuity, etc.) as $h(t)$. This is proved by solving (3.2) iteratively.

LEMma 2. (a) $u(t) \in D(A)$ and $u(t), A(t) u(t)$ are continuous for $0 \leqq t \leqq t_{0}$ (for some $\left.t_{0}<T\right)$ if and only if the same is true of $\tilde{u}(t)$.

(b) $u(t) \in D(A), u(t)$ is continuous for $0 \leqq t \leqq t_{0}, A(t) u(t)$ is continuous for $0<t \leqq t_{0}$ and $\|A(t) u(t)\|$ is integrable in $\left(0, t_{0}\right)$ if and only if the same is true of $\tilde{u}(t)$.

Proof. Let $u$ be as in (a). Then $\tilde{u}(t)$ is continuous in [0, $\left.t_{0}\right]$. Write

$$
A(t) u(\tau)=\left\{I+[A(t)-A(\tau)] A^{-1}(\tau)\right\} A(\tau) u(\tau) .
$$

$A(t) A^{-1}(\tau)$ is continuous in $\tau$ (it is, in fact, Hölder continuous) since the same is true of its inverse (by (1.2)). Therefore $A(t) u(\tau)$ is continuous in $\tau$. Hence the integral $\int_{0}^{t} h(t-\tau) A(t) u(\tau) d \tau$ exists. Since the integral $\int_{0}^{t} h(t-\tau) u(\tau) d \tau$ also exists and since $A(t)$ is a closed operator, we conclude that the latter integral belongs to $D(A)$, and

$$
A(t) \int_{0}^{t} h(t-\tau) u(\tau) d \tau=\int_{0}^{t} h(t-\tau) A(t) u(\tau) d \tau .
$$

The last integral is continuous for $0 \leqq t \leqq t_{0}$. To prove this, write

$$
A\left(t^{\prime}\right) u(\tau)-A(t) u(\tau)=\left\{\left[A\left(t^{\prime}\right)-A(t)\right] A^{-1}(t)\right\} A(t) u(\tau)
$$

and observe that the operator in braces is bounded (cf. (1.2)) by $C^{\prime}\left|t^{\prime}-t\right|^{\alpha}$, whereas $A(t) u(\tau)$ is continuous in $\tau$.

We conclude from (3.2) that $\tilde{u}(t) \in D(A)$ and $A(t) \tilde{u}(t)$ is continuous for $0 \leqq t \leqq t_{0}$.

To prove the second part of (a) we use (3.5) and argue as before. The proof of (b) is similar.

We shall also need the following lemma.

LEMMA 3. For any $0<\mu<1$ there exist positive constants $H_{1}, H_{2}$ such that for 
any continuous function $u(t)\left(0 \leqq t \leqq t_{0}\right)$ with $A(t) u(t)$ continuous for $0<t \leqq t_{0}$ and $\|A(t) u(t)\|$ integrable in $\left(0, t_{0}\right)$,

$$
\begin{gathered}
H_{1} \sup _{0<t<t_{0}}\|u(t)\| \leqq \sup _{0<t<t_{0}}\|\tilde{u}(t)\| \leqq H_{2} \sup _{0<t<t_{0}}\|u(t)\|, \\
H_{1} \sup _{0<t<t_{0}} t^{\mu}\|A(t) u(t)\| \leqq \sup _{0<t<t_{0}} t^{\mu}\|A(t) \tilde{u}(t)\| \leqq H_{2} \sup _{0<t<t_{0}}\left\|t^{\mu} A(t) u(t)\right\| .
\end{gathered}
$$

The constants $H_{1}, H_{2}$ are independent of $t_{0}$.

Note that, by Lemma $2, A(t) \tilde{u}(t)$ is well defined.

Proof. (3.8) follows immediately from (3.2), (3.5). To prove the second inequality in (3.9), apply $t^{\mu} A(t)$ to (3.2). Using (3.6), (1.2) we get

$$
\left\|\int_{0}^{t} h(t-\tau) t^{\mu} A(t) u(\tau) d \tau\right\| \leqq \text { const } \sup _{0<\tau<t} \tau^{\mu}\|A(\tau) u(\tau)\| .
$$

Therefore,

$$
\left\|t^{\mu} A(t) \tilde{u}(t)\right\| \leqq\left\|t^{\mu} A(t) u(t)\right\|+\underset{0<\tau<t}{\text { const }} \tau^{\mu}\|A(\tau) u(\tau)\|,
$$

from which the desired inequality follows. The first inequality in (3.9) follows by applying the same argument to (3.5).

We now return to the system (3.3). Denoting by $U(t, \tau)$ the fundamental solution of the equation $d v / d t+h(0) A(t) v=0$, we represent $\tilde{u}$, formally, in the form:

$$
\tilde{u}(t)=U(t, 0) k(0)+\int_{0}^{t} U(t, s) \dot{k}(s) d s+\int_{0}^{t} U(t, s) f(\tilde{u} ; s) d s .
$$

Definition. A function $\tilde{u}(t)$ defined for $0 \leqq t \leqq t_{0}$ is called a weak solution of (3.3) in $\left[0, t_{0}\right]$ if it is continuous in $\left[0, t_{0}\right]$, if $\tilde{u}(t) \in D(A), A(t) \tilde{u}(t)$ is continuous in $\left(0, t_{0}\right]$ and $\|A(t) \tilde{u}(t)\|$ is integrable in $\left(0, t_{0}\right)$, and, finally, if (3.10) holds for $0 \leqq t \leqq t_{0}$, with $f(\tilde{u} ; t)$ given by (3.4).

LEMMA 4. $\tilde{u}$ is a weak solution of (3.3) in $\left[0, t_{0}\right]$ for some $t_{0}<T$, if and only if $u$ is a strong solution of (1.4).

Proof. Suppose $u$ is a strong solution of (1.4). Then, by Lemma 2(b), $\tilde{u}$ satisfies all the properties of a weak solution except possibly (3.10). From (1.4) it is clear that $u(t)$ is uniformly Hölder continuous in $\left[0, t_{0}\right]$, that $d u(t) / d t$ exists for $0<t \leqq t_{0}$, and that (3.1) holds. To go from (3.1) to (3.3) we observe that since $h(t)$ is absolutely continuous, the same is true of $\int_{0}^{t} h(t-\tau) u(\tau) d \tau$, and the derivative of this integral is almost everywhere equal to

$$
h(0) u(t)+\int_{0}^{t} \ddot{h}(t-\tau) u(\tau) d \tau
$$

Since, further, $u(t)$ is absolutely continuous in any interval $\left(\varepsilon, t_{0}\right)$ with $\varepsilon>0$, the same is true of $\tilde{u}(t)$. We conclude that (3.3) holds almost everywhere. We shall 
prove in a moment that $f(\tilde{u} ; t)$ is continuous (in fact Hölder continuous). Once this is done, we shall have that all the terms in (3.3) are continuous for $0<t \leqq t_{0}$, with the possible exception of $d \tilde{u} / d t$. It will therefore follow that $d \tilde{u} / d t$ exists everywhere in $\left(0, t_{0}\right]$ and satisfies $(3.3)$.

Once we have proved that $f(\tilde{u} ; t)$ is also uniformly Hölder continuous in $t$ in $\left[0, t_{0}\right]$, then it will follow from (3.3) that $\tilde{u}$ satisfies (3.10) and is thus a weak solution of (3.3).

Now, using (3.7) and (1.2), and noting (by (3.6), (1.2)) that $\|A(t) u(\tau)\|$ is integrable in $\tau$, we easily conclude that the first integral on the right-hand side of (3.4) is uniformly Hölder continuous in $\left[0, t_{0}\right]$. The same is true of the last integral in (3.4), since it is equal to $\int_{0}^{t} \vec{h}(\tau) u(t-\tau) d \tau$ and $u(t)$ is uniformly Hölder continuous in $\left[0, t_{0}\right]$, whereas $\vec{h}(t)$ is in $L^{p}(0, T)$. It follows that $f(\tilde{u} ; t)$ is uniformly Hölder continuous in $\left[0, t_{0}\right]$.

Suppose conversely that $\tilde{u}$ is a weak solution of (3.3). Then $u$ satisfies all the properties of a strong solution, except possibly (1.4) and the condition that $t^{\delta}\|A(t) u(t)\| \leqq$ const for some $0 \leqq \delta<1$. We have, by (2.8) (with $\rho=0, \sigma=\mu$ ),

$$
\begin{aligned}
\|U(t+\Delta t, 0) k(0)-U(t, 0) k(0)\| & \leqq\left\|[U(t+\Delta t, 0)-U(t, 0)] A^{-\mu}(0)\right\|\left\|A^{\mu}(0) k(0)\right\| \\
& \leqq \text { const }|\Delta t|^{\mu}
\end{aligned}
$$

Thus, the first term on the right-hand side of (3.10) is uniformly Hölder continuous with exponent $\mu$. The same is true also of the two integrals in (3.10). Hence $\tilde{u}(t)$ is uniformly Hölder continuous with exponent $\mu$, and the same is then true of $u$.

As in the first part of the proof we can now show that $f(\tilde{u} ; t)$ is uniformly Hölder continuous in $\left[0, t_{0}\right]$. Consequently, (3.10) is equivalent to (3.3). By an argument similar to that given in the first part we conclude that $d u / d t$ exists and (3.1) is satisfied everywhere in $\left(0, t_{0}\right]$. Since (3.1) is the differentiated form of (1.4), (1.4) follows.

It remains to prove that $t^{\delta}\|A(t) u(t)\| \leqq$ const for some $0 \leqq \delta<1$. From (2.7) (with $\rho=1, \sigma=\mu$ ) we get

$$
\|A(t) U(t, 0) k(0)\| \leqq\left\|A(t) U(t, 0) A^{-\mu}(0)\right\|\left\|A^{\mu}(0) k(0)\right\| \leqq \text { const } t^{\mu-1} .
$$

The same bound is easily obtained for the other terms on the right-hand side of (3.10). Hence

$$
\|A(t) \tilde{u}(t)\| \leqq \text { const } t^{\mu-1} \text {. }
$$

By Lemma 3, the same inequality holds for $\|A(t) u(t)\|$.

From the proof of the second part of Lemma 4 we also obtain the uniform Hölder continuity of $u(t)$ with exponent $\mu$, and the boundedness of $t^{1-\mu}\|A(t) u(t)\|$ for $t \rightarrow 0$, as asserted in the last part of Theorem 1. If $k(0) \in D(A)$, then $A(t) U(t, 0) k(0)$ is continuous in $\left[0, t_{0}\right]$, and $A(t) \tilde{u}(t)$ is then also continuous in $\left[0, t_{0}\right]$. By Lemma 2(a), the same is true of $A(t) u(t)$. Since $d u(t) / d t$ is then clearly continuous in $\left[0, t_{0}\right]$ also, the proof of the last part of Theorem 1 is complete. 
We proceed to prove existence and uniqueness of a weak solution of (3.3). For simplicity, we first impose the conditions:

$$
h(0)=0 \text {, }
$$

(3.13) $h \in C^{2}[0, T)$ and $\ddot{h}(t)$ is uniformly Hölder continuous in $[0, T)$.

The proof is given by a step-by-step procedure. The result for the first step is stated in the following lemma.

LEMMA 5. If $t_{0}$ is sufficiently small then there exists a unique weak solution of (3.3) in $\left(0, t_{0}\right]$.

Proof. Without loss of generality we may assume that $\mu<1$. Denote by $B_{0}$ the set of all functions $v(t)$ from $\left[0, t_{0}\right]$ into $X$ satisfying: $v(t) \in D(A), v(t)$ is continuous in $\left[0, t_{0}\right], A(t) v(t)$ is continuous in $\left(0, t_{0}\right]$ and $t^{1-\mu}\|A(t) v(t)\|$ is a bounded function. Introduce in $B_{0}$ the norm

$$
\|v\|=\sup _{0<t<t_{0}}\|v(t)\|+\sup _{0<t<t_{0}} t^{1-\mu}\|A(t) v(t)\| .
$$

Since $A(t)$ is a closed operator, $B_{0}$ is easily seen to be a Banach space. Set $B_{0 M}=\left\{v \in B_{0} ;\|v\| \leqq M\right\}$.

Denote the right-hand side of (3.10) by $S \tilde{u}$ and the sum of the first two terms on the right-hand side of (3.10) by $\tilde{k}(t)$. Set $S \tilde{u}=\tilde{k}+S_{0} \tilde{u}$. Using (2.7) we find that $\tilde{k} \in B_{0}$. Next, by arguments given in the proof of Lemma 4 it follows that if $\tilde{u} \in B_{0}$ then $S_{0} \tilde{u} \in B_{0}$. If we prove that for some $M>0, S$ maps $B_{0 M}$ into itself and is a contraction, then it will follow that $S$ has a unique fixed point in $B_{0 M}$, i.e., a unique solution $\tilde{u} \in B_{0 M}$ of (3.10).

Now, by Lemmas 2 and $3, u \in B_{0}$ if and only if $\tilde{u} \in B_{0}$ and

$$
\text { const }\|\tilde{u}\| \leqq\|u\| \leqq \text { const }\|\tilde{u}\|,
$$

where the constants are positive. Using (1.2), (3.12), (3.13), (3.15), we get from (3.4),

$$
\begin{gathered}
\|f(\tilde{u} ; t)\| \leqq \text { const } t^{\delta}\|\tilde{u}\| \quad(\delta=\min (1, \alpha+\mu)), \\
\|f(\tilde{u} ; t)-f(\tilde{u} ; s)\| \leqq \text { const } t^{\mu}|t-s|^{\alpha}\|\tilde{u}\| \quad\left(0 \leqq s \leqq t \leqq t_{0}\right) .
\end{gathered}
$$

Applying Lemma 1, we get

Since

$$
t^{1-\mu}\left\|A(t) S_{0} \tilde{u}(t)\right\| \leqq \text { const } t\|\tilde{u}\| .
$$

we get

$$
\left\|S_{0} \tilde{u}(t)\right\| \leqq \text { const } t^{1+\delta}\|\tilde{u}\|
$$

$S_{0}$ is a linear operator; we conclude that if $2 L t_{0} \leqq 1$ then $S_{0}$, and therefore also $S$, are contraction operators. If further $M$ is such that $2\|\tilde{k}\| \leqq M$ then $S$ maps $B_{0 M}$ into itself. 
We have thus proved the existence and uniqueness of a weak solution of (3.3) in $B_{0 M}$. If $v$ is any other weak solution in $\left[0, t_{0}\right]$ then, as is easily seen, $v$ must belong to $B_{0}$. Hence $w \equiv \tilde{u}-v$ is a solution with $\tilde{k} \equiv 0$. But then

$$
\|w\|=\|S w\|=\left\|S_{0} w\right\|<\|w\|, \quad \text { if } w \not \equiv 0,
$$

which is impossible; hence $\tilde{u} \equiv v$.

LEMMA 6. There exists a unique weak solution of (3.3) in $[0, T-\varepsilon]$ for any $\varepsilon>0$.

Proof. We first prove the assertion in an interval $\left[0, t_{1}\right]$ for some $t_{1}>t_{0}$, with $t_{1}-t_{0}$ sufficiently small. We define a space $B_{1}$ in the same way as $B_{0}$, except that $t_{0}$ is replaced by $t_{1}$. The definition of $B_{1 N}$ is significantly different from that of $B_{0 N}$, namely, $B_{1 N}$ consists of all the functions $v(t)$ satisfying $\|v\| \leqq N$ and coinciding with the solution $u(t)$ for $0 \leqq t \leqq t_{0}$. Thus, if $0 \leqq t \leqq t_{0}$ then $S v=S \tilde{u}=\tilde{u}$, whereas if $t_{0}<t \leqq t_{1}$ then $S v=\tilde{k}_{1}+S_{1} v$ where

$$
\begin{array}{rlrl}
\tilde{k}_{1}(t) & =k(t)+\int_{0}^{t_{0}} U(t, s) f(\tilde{u} ; s) d s & \left(t_{0}<t \leqq t_{1}\right), \\
S_{1} v(t) & =\int_{t_{0}}^{t} U(t, s) f(\tilde{v} ; s) d s & & \left(t_{0}<t \leqq t_{1}\right) .
\end{array}
$$

$S_{1} v$ can be estimated entirely analogously to $S_{0} v$. We obtain:

$$
\begin{aligned}
\|S v\| & \leqq M_{1}+L_{1}\left(t_{1}-t_{0}\right)\|v\|, \\
\|S v-S \hat{v}\| & \leqq L_{1}\left(t_{1}-t_{0}\right)\|v-\hat{v}\|,
\end{aligned}
$$

where $M_{1}$ is a constant depending on $k, M$ and $L_{1}$ is a constant. Hence, if $N=2 M_{1}$ and $2 L_{1}\left(t_{1}-t_{0}\right) \leqq 1$ then $S$ maps the closed bounded set $B_{1 N}$ into itself and is a contraction. Consequently $S$ has a unique fixed point, i.e., a unique weak solution of (3.3) in $\left[0, t_{1}\right]$ in $B_{1 N}$. This solution coincides with $\tilde{u}(t)$ in $\left[0, t_{0}\right]$. As in the proof of the previous lemma, one shows that there cannot exist any other weak solution of (3.3) in $\left[0, t_{1}\right]$.

We can now proceed in this way step-by-step to $\left[0, t_{3}\right],\left[0, t_{4}\right]$, etc. Noting that the constant $L_{1}$ can be taken to be the same for each step, the assertion of the lemma follows.

To complete the proof of Theorem 1 it remains to remove the restrictions (3.12), (3.13). We begin with (3.12). Introducing the functions $u_{0}=u e^{-\varepsilon t}, \tilde{u}_{0}=\tilde{u} e^{-\varepsilon t}$ we see that (1.4), (3.1)-(3.4) hold for this new pair of functions provided $k(t), \dot{k}(t)$, $d^{j} h(t) / d t^{j}(j=0,1,2)$ are replaced by $k(t) e^{-\varepsilon t}, \dot{k}(t) e^{-\varepsilon t},\left(d^{j} h(t) / d t^{j}\right) e^{-\varepsilon t}$, respectively, and provided we add a term $-\varepsilon u_{0}(t)$ on the right-hand sides of (3.1) and (3.4). Hence, if we take $\varepsilon=h(0) / h(0),(3.12)$ will be satisfied. Since Lemmas 3, 4 remain true for the new equations, we get the existence and uniqueness of a strong solution of the equation obtained from (1.4) by the above transformation. The assertion for (1.4) then immediately follows. 
We give another way of removing the restriction (3.12) which is more involved but which has the advantage that it removes also the restriction (3.13). One modifies the definition of $B_{0}$ by imposing upon its functions the condition that they satisfy a uniform Hölder condition with some fixed exponent $\eta \leqq \mu$. The definition of $\|v\|$ in (3.14) is modified by adding on the right-hand side the Hölder coefficient of $v$. We can then proceed analogously to the proof of Lemma 5, making use also of (3.11) and (2.8).

Now, if the condition (3.13) is not satisfied then in extending the proof of (3.17) we obtain the desired bound on the Hölder coefficient of the last integral occurring in the definition (3.4) of $f(\tilde{u} ; t)$ by writing it in the form $\int_{0}^{t} \ddot{h}(\tau) u(t-\tau) d \tau$ and using the Hölder continuity of $u(t)$. In treating the first integral on the right-hand side of (3.4) we use the uniform Hölder continuity of $h(t)$ with exponent $1 / q$, where $1 / p+1 / q=1$. The right-hand side of (3.17) is replaced by

$$
\text { const }|t-s|^{v}\|\tilde{u}\| \| \quad \text { where } \nu=\min (\eta, \alpha, 1 / q) \text {. }
$$

(3.18) holds with $t_{0}$ replaced by $t_{0}^{1-\mu}$, and with obvious modifications the proof of Lemma 5 can be completed. The proof of Lemma 6 remains unchanged.

Proof of Corollary. It suffices to prove that any solution of (1.4) is necessarily a strong solution. We can clearly restrict ourselves to an interval $0 \leqq t \leqq \varepsilon$ with arbitrarily small $\varepsilon>0$. In view of Lemma 4 it suffices to show that any solution of (1.4) is a weak solution. Following the proof of the first part of Lemma 4 we see that all we have to show is that $f(\tilde{u} ; t)$ is uniformly Hölder continuous. Now, the Hölder continuity of the first integral on the right-hand side of (3.4) is proved as before; the Hölder continuity of the last integral in (3.4) is a consequence of our additional assumption on $h(t)$. Finally, we may assume that $h(0)=0$ since otherwise we perform the reduction to this case described in the proof of Theorem 1 . Thus, $f(\tilde{u} ; t)$ is in fact uniformly Hölder continuous.

\section{Proofs of Theorems $2,4$.}

Proof of Theorem 2. Proceeding analogously to the proof of Theorem 1 we first reduce (1.6), formally, to the system

where

$$
\begin{aligned}
\frac{d \tilde{V}}{d t}+h(0) A(t) \tilde{V} & =F(\tilde{V} ; t), \\
\tilde{V}(s) & =A^{-\mu}(s),
\end{aligned}
$$

$$
\begin{aligned}
\tilde{V} ; t F()= & \int_{s}^{t} h(t-\tau)\left\{[A(t)-A(\tau)] A^{-1}(\tau)\right\} A(\tau) V(\tau) d \tau \\
& +\frac{h(0)}{h(0)} V(t)+\frac{1}{h(0)} \int_{s}^{t} \ddot{h}(t-\tau) V(\tau) d \tau
\end{aligned}
$$

and where we have set $V(t)=W_{\mu}(t, s)$ and

$$
\tilde{V}(t)=V(t)+\frac{1}{h(0)} \int_{s}^{t} h(t-\tau) V(\tau) d \tau .
$$


The analog of $(3.10)$ is

$$
\tilde{V}(t)=U(t, s) A^{-\mu}(s)+\int_{s}^{t} U(t, \tau) F(\tilde{V} ; \tau) d \tau .
$$

We can now proceed to establish analogs of Lemmas 1-6. Instead of the functions $u(t)$ with values in $X$ we now deal with analogous functions with values in $B(X)$ (the Banach space of bounded operators on $X$ ). The analog of $k(t)$ is the constant operator $A^{-\mu}(s)$. Since all the considerations are very similar to those of $\S 1.3$, we omit further details.

It remains to prove that $W_{\mu}(t, s)$ is strongly continuous in $(t, s)$ for $0 \leqq s \leqq t<T$. This is true of $U(t, s)$, and also of $A^{-\mu}(s)$ (in fact, by [15, p. 316],

$$
\left.\left\|A^{-\mu}(s)-A^{-\mu}\left(s^{\prime}\right)\right\| \leqq \text { const }\left|s-s^{\prime}\right|^{\alpha}\right) .
$$

Hence, $B(t, s) \equiv U(t, s) A^{-\mu}(s)$ is continuous in $(t, s)$. Recalling that the transformation $S_{0} \tilde{V}$ defined by the integral in (4.4) has norm less than $1 / 2$ if $t_{0}$ is sufficiently small, and verifying that $t_{0}$ can be taken to be independent of $s$, we can write

$$
W_{\mu}(t, s)=B(t, s)+\sum_{n=1}^{\infty}\left(S_{0}^{n} B\right)(t, s)
$$

Since each of the integrals $\left(S_{0}^{n} B\right)(t, s)$ is absolutely convergent and each integrand is continuous in $(t, s)$ for $0 \leqq s \leqq t \leqq t_{0}$, each term in the series of (4.5) is continuous in $(t, s)$. Since, finally, the series in (4.5) is uniformly convergent (in fact, $\left\|S_{0}^{n} B(t, s)\right\|$ $\left.\leqq 1 / 2^{n}\right)$, the continuity of $W_{\mu}(t, s)$ in $0 \leqq s \leqq t \leqq t_{0}$ follows. To derive the continuity in $[0, T)$, we proceed step-by-step on $t$-intervals (compare the proof of Lemma 6).

Proof of Theorem 4. Substituting (1.9) into (1.4) we get

$$
W_{\mu}(t, 0) A^{\mu}(0) k(0)+\int_{0}^{t} W_{\mu}(t, s) A^{\mu}(s) \dot{k}(s) d s=k(t)
$$

$$
-\int_{0}^{t} h(t-\tau) A(\tau) W_{\mu}(\tau, 0) A^{\mu}(0) k(0) d \tau-\int_{0}^{t} h(t-\tau)\left\{\int_{0}^{\tau} A(\tau) W_{\mu}(\tau, s) A^{\mu}(s) \dot{k}(s) d s\right\} d \tau .
$$

By (1.7) it is permissible to change the order of integration in the last term. We find that it is equal to

$$
\begin{array}{r}
-\int_{0}^{t}\left\{\int_{s}^{t} h(t-\tau) A(\tau) W_{\mu}(\tau, s) d \tau\right\} A^{\mu}(s) \dot{k}(s) d s \\
=-\int_{0}^{t} \dot{k}(s) d s+\int_{0}^{t} W_{\mu}(t, s) A^{\mu}(s) \dot{k}(s) d s .
\end{array}
$$

Hence, (4.6) is equivalent to

$$
W_{\mu}(t, 0) A^{\mu}(0) k(0)=k(0)-\int_{0}^{t} h(t-\tau) A(\tau) W_{\mu}(\tau, 0) A^{\mu}(\tau) k(0) d s .
$$

But this relation follows by applying (1.6), with $s=0$, to $A^{\mu}(0) k(0)$. 
We have thus proved that the function $u(t)$, given by the right-hand side of (1.9), is a solution of (1.4). Since by (1.7) $\|A(t) u(t)\|$ is bounded by const $t^{\mu-1}$ as $t \rightarrow 0$, $u(t)$ is also a strong solution. Consequently, it coincides with the solution whose existence is asserted in Theorem 1 .

5. Further properties of $W$. We have not proved so far that $W(t, s)$ is a bounded operator. This we shall do in this section, but only under an additional condition on the $A(t)$. Our method is to rewrite (1.5) in a "weak" form and prove that this equation has a solution which is a bounded operator function. Then we show that this solution coincides with the fundamental solution of $\S 1$. In the following condition $\rho$ is a number satisfying: $0 \leqq \rho<1$.

$\left(\mathrm{B}_{\rho}\right)$ The domain $D\left(A^{\rho}\right)$ of $A^{\rho}(t)$ is independent of $t$, and for any $t, \tau$ in $[0, T)$,

$$
\left\|[A(t)-A(\tau)] A^{-\rho}(\tau)\right\| \leqq C^{\prime}|t-\tau|^{\alpha}
$$

where $C^{\prime}, \alpha$ are positive constants, and $0<\alpha \leqq 1$.

Note that $\left(\mathbf{B}_{\rho}\right)$ implies $(\mathbf{B})$.

If $\left(\mathrm{B}_{\rho}\right)$ holds, then we can rewrite the function $f(\tilde{u} ; t)$ of $(3.4)$ in the form

$$
\begin{aligned}
f_{\rho}(\tilde{u} ; t)= & \int_{0}^{t} h(t-\tau)\left\{[A(t)-A(\tau)] A^{-\rho}(\tau)\right\} A^{\rho}(\tau) u(\tau) d \tau \\
& +\frac{h(0)}{h(0)} u(t)+\frac{1}{h(0)} \int_{0}^{t} \ddot{h}(t-\tau) u(\tau) d \tau .
\end{aligned}
$$

For the sake of reference we also rewrite (3.3), (3.10):

$$
\begin{gathered}
\frac{d \tilde{u}}{d t}+h(0) A(t) \tilde{u}=\dot{k}(t)+f_{\rho}(\tilde{u} ; t), \\
\tilde{u}(0)=k(0), \\
\tilde{u}(t)=U(t, 0) k(0)+\int_{0}^{t} U(t, s) \dot{k}(s) d s+\int_{0}^{t} U(t, s) f_{\rho}(\tilde{u} ; s) d s .
\end{gathered}
$$

Lemmas 2, 3 remain true if $A(t)$ is replaced everywhere by $A^{\rho}(t)$.

Definition. $u$ is a $\rho$-weak solution of $(1.4)$ in $\left[0, t_{0}\right]$ if $\tilde{u}(t)$ is defined and continuous for $0 \leqq t \leqq t_{0}, \tilde{u}(t) \in D\left(A^{\rho}\right)$ and $A^{\rho}(t) \tilde{u}(t)$ is continuous for $0<t \leqq t_{0}$, if $\left\|A^{\rho}(t) \tilde{u}(t)\right\|$ is integrable in $\left(0, t_{0}\right)$ and, finally, if (5.4) holds. If $u$ is a $\rho$-weak solution of (1.4) then we also say that $\tilde{u}$ is a $\rho$-weak solution of (5.3) or of (5.4).

If $u$ is a strong solution then $\tilde{u}$ is obviously a $\rho$-weak solution since (see, for instance, [15])

$$
\left\|A^{\rho}(t) v\right\| \leqq \text { const }\|A(t) v\|^{\rho}\|v\|^{1-\rho} .
$$

Conversely, if $\tilde{u}$ is a $\rho$-weak solution then we can proceed analogously to the proof of the second part of Lemma 4 and show that $f_{\rho}(\tilde{u} ; t)$ is uniformly Hölder continuous in $\left[0, t_{0}\right]$. From (5.4) it also follows that $\|A(t) u(t)\| \leqq$ const $t^{u-1}$. The proof that $u$ is a strong solution is then easily completed. 
We have thus established:

LEMma 7. Assume that (A), $\left(\mathrm{B}_{\rho}\right),(\mathrm{H}),(\mathrm{K})$ hold. Then $u$ is a strong solution of (1.4) if and only if $u$ is a $\rho$-weak solution of (1.4).

The proof of Lemmas 5, 6 can be modified to prove the existence and uniqueness of a $\rho$-weak solution directly. Thus, instead of the norm (3.14) we now use the norm

$$
\|v\|=\sup _{0<t<t_{0}}\|v(t)\|+\sup _{0<t<t_{0}} t^{\rho-\mu}\left\|A^{\rho}(t) v(t)\right\| .
$$

Furthermore, in deriving the analog of (3.18) there is no need to use Lemma 1.

The above considerations will next be extended to operator-valued functions. We say that $W(t, s)$ is a $\rho$-weak solution of $(1.5)$ in $[0, T)$ if $W(t, s)$ is strongly continuous in $(t, s)$ for $0 \leqq s \leqq t<T$, if $W(t, s) \in D\left(A^{\rho}\right)$ and $A^{o}(t) W(t, s)$ is strongly continuous in $t$ for $s<t<T$, if $\left\|A^{\rho}(t) W(t, s)\right\|$ is integrable in $t$ in any interval $(s, T-\varepsilon)$ with $\varepsilon>0$, and, finally, if

$$
\tilde{V}(t)=U(t, s)+\int_{s}^{t} U(t, \tau) F_{\rho}(\tilde{V} ; \tau) d \tau
$$

where

$$
\begin{aligned}
F_{\rho}(\tilde{V} ; t)= & \int_{s}^{t} h(t-\tau)\left\{[A(t)-A(\tau)] A^{-\rho}(\tau)\right\} A^{\rho}(\tau) V(\tau) d \tau \\
& +\frac{h(0)}{h(0)} V(t)+\frac{1}{h(0)} \int_{s}^{t} \ddot{h}(t-\tau) V(\tau) d \tau,
\end{aligned}
$$

$V(t)=W(t, s)$ and $\tilde{V}(t)$ is defined by (4.3).

Similarly, one can define a $\rho$-weak solution of (1.6). By arguments similar to those given above, it follows that $W_{\mu}(t, s)$ is a strong solution of $(1.6)$ if and only if $W_{\mu}(t, s)$ is a $\rho$-weak solution of (1.6).

We shall need the following condition on $h$ :

$\left(\mathrm{H}^{\prime}\right) h(0)>0, h \in C^{2}[0, T)$ and $\breve{h}(t)$ is uniformly Hölder continuous in closed subsets of $[0, T)$.

THEOREM 5. Assume that $(\mathrm{A}),\left(\mathrm{B}_{\rho}\right),\left(\mathrm{H}^{\prime}\right)$ hold. Then there exists a unique $\rho$-weak solution $W(t, s)$ of $(1.5)$ in $[0, T)$. It coincides with the fundamental solution of $\S 1$ and it satisfies:

$$
\left\|A^{\rho}(t) W(t, s)\right\| \leqq \operatorname{const}(t-s)^{-\rho} .
$$

The proof is similar to the proof of existence and uniqueness of a $\rho$-weak solution of (5.3), as outlined above. The condition $\left(\mathrm{H}^{\prime}\right)$ arises from the need to have the condition (3.13) fulfilled. One cannot dispose of the condition (3.13) as in the case of (5.3). The proof of continuity of $W(t, s)$ in $(t, s)$ is similar to the corresponding proof for $W_{\mu}(t, s)$ in $\S 1.4$. 
Finally, if $x \in D\left(A^{\mu}\right)$ then $v(t) \equiv W(t, s) x$ is easily seen to be a $\rho$-weak solution of

$$
v(t)=x-\int_{s}^{t} h(t-\tau) A(\tau) v(\tau) d \tau
$$

Hence, by Theorem 3 and Lemma $7, W(t, s)$ coincides with the fundamental solution of $\S 1$.

Corollary 1. $\partial W(t, s) / \partial t$ and $A(t) W(t, s)$ are continuous for $s<t<T$, and $\tilde{W}(t, s)$ satisfies the equation

$$
\partial \tilde{W}(t, s) / \partial t+h(0) A(t) \tilde{W}(t, s)=F_{\rho}(\tilde{W} ; t) \quad(s<t<T)
$$

where $F_{\rho}$ is defined in (5.7) with $V(t)=W(t, s)$.

Proof. In fact, if $h(0)=0$ then this follows by arguments used in the proof of the second part of Lemma 4 . The restriction $h(0)=0$ can be removed by using a transformation $W \rightarrow W e^{-\varepsilon t}$ with $\varepsilon=h(0) / h(0)$.

COROLlary 2. If in Theorem 4 we also assume that the conditions $\left(\mathrm{B}_{\rho}\right),\left(\mathrm{H}^{\prime}\right)$ hold, then the assertion is valid for any function $k(t)$ satisfying $(\mathrm{K})$.

Proof. By linearity, it suffices to prove the corollary for each of the functions $k(0)$ and $k(t)-k(0)$. Since the assertion for $k(0)$ follows from Theorem 4 , we can now restrict ourselves to functions $k(t)$ with $k(0)=0$. For the function $u(t)$ given by the right-hand side of (1.9), one can easily verify that

$$
\begin{aligned}
\tilde{u}(t) & =\int_{0}^{t} \tilde{W}(t, s) \dot{k}(s) d s, \\
f_{\rho}(\tilde{u} ; t) & =\int_{0}^{t} F_{\rho}(\tilde{W} ; t) \dot{k}(s) d s .
\end{aligned}
$$

Substituting (1.9) into (5.4) and using the last two relations and (5.6), we find that (5.4) holds. Since also $\left\|A^{\rho}(t) u(t)\right\|$ is integrable (by (5.8)), $u$ is a $\rho$-weak solution of (1.4). By Lemma 7 it is also a strong solution, and thus it coincides with the solution of Theorem 1 .

In concluding this section we mention that various bounds which hold for $U(t, s)$ can be derived also for $W(t, s)$. For instance,

$$
\|A(t) W(t, s)\| \leqq \operatorname{const}(t-s)^{-1} \text {. }
$$

The proof of (5.10) in case $h(0)=0$ follows immediately from (5.6), (5.8) and the uniform Hölder continuity of $F(\tilde{V} ; t)$. If $h(0) \neq 0$ then we perform a transformation $W \rightarrow W e^{-\varepsilon t}$ with $\varepsilon=h(0) / h(0)$.

Analogs of (2.7), (2.8) can also be derived.

6. Global bounds on $W$. In this section, we assume that the conditions (A), $\left(\mathrm{B}_{\rho}\right),\left(\mathrm{H}^{\prime}\right)$ hold with $T=\infty$. We set

$$
\tilde{A}(t)=h(0) A(t)-(h(0) / h(0)) I
$$


and denote by $V(t, s)$ the fundamental solution of the equation $d v / d t+\tilde{A}(t) v=0$. Then, as is easily verified, $V(t, s)=e^{\nu(t-s)} U(t, s)$, where $\nu=h(0) / h(0)$ and $U(t, s)$ is the fundamental solution of $d v / d t+h(0) A(t) v=0$. Since the inequality

$$
\left\|A^{\lambda}(t) U(t, s)\right\| \leqq K(t-s)^{-\lambda} e^{a(t-s)}
$$

is valid for any $0 \leqq \lambda \leqq 1$ and positive constants $K, a$ (in fact this follows from [15] by estimating successively the terms of $\Phi$ in formula (1.39) of [15]), we also get

$$
\left\|A^{\lambda}(t) V(t, s)\right\| \leqq K(t-s)^{-\lambda} e^{a(t-s)} \quad(0 \leqq \lambda \leqq 1)
$$

with different positive constants $K, a$.

Our purpose is to obtain a similar estimate for $W(t, s)$. We shall need the following assumption:

$$
|h(t)|+|\ddot{h}(t)| \leqq K^{\prime} e^{b t}
$$

where $K^{\prime}, b$ are positive constants.

In what follows, various positive constants which are independent of $t, s, \tau$, $\sigma$ will be denoted by $K$ or by $c$.

THEOREM 6. Assume that (A), $\left(\mathrm{B}_{\rho}\right),\left(\mathrm{H}^{\prime}\right),(6.4)$ hold with $T=\infty$. Then, for $0 \leqq s<t<\infty$,

$$
\left\|A^{\lambda}(t) W(t, s)\right\| \leqq K(t-s)^{-\lambda} e^{c(t-s)} \quad \text { for any } 0 \leqq \lambda \leqq \rho .
$$

Proof. Writing (5.9) in the form

$$
\partial \tilde{W}(t, s) / \partial t+\tilde{A}(t) \tilde{W}(t, s)=\tilde{F}_{\rho}
$$

and using (5.7), we can represent $\tilde{W}(t, s)$ in terms of the fundamental solution $V(t, \tau)$ :

$$
\begin{gathered}
\tilde{W}(t, s)=V(t, s)+\int_{s}^{t} V(t, \sigma)\left\{\int_{s}^{\sigma} h(\sigma-\tau)\left\{[A(\sigma)-A(\tau)] A^{-\rho}(\tau)\right\} A^{\rho}(\tau) W(\tau, s) d \tau\right\} d \sigma \\
+\int_{s}^{t} V(t, \sigma)\left\{\int_{s}^{\sigma} \tilde{h}(\sigma-\tau) W(\tau, s) d \tau\right\} d \sigma
\end{gathered}
$$

where

$$
\tilde{h}(t)=\frac{\ddot{h}(t)}{h(0)}-\frac{h(t)}{h^{2}(0)}
$$

Applying $A^{\rho}(t)$ to $(4.3)$ with $V(t)=W(t, s)$ we get

$$
\begin{aligned}
& A^{\rho}(t) W(t, s) \\
& \quad=A^{\rho}(t) \tilde{W}(t, s)+\frac{1}{h(0)} \int_{s}^{t} h(t-\tau)\left\{I+\left[A^{\rho}(t)-A^{\rho}(\tau)\right] A^{-\rho}(\tau)\right\} A^{\rho}(\tau) W(\tau, s) d \tau .
\end{aligned}
$$

Writing $\phi(t, s)=\left\|A^{\rho}(t) W(t, s)\right\|$, substituting $\tilde{W}(t, s)$ from (6.6) into (6.7) and using (6.3) (with $\lambda=\rho$ ), (6.4) and the inequality

$$
\|W(\tau, s)\| \leqq K\left\|A^{\rho}(\tau) W(\tau, s)\right\|
$$


(which follows from the boundedness of $A^{-\rho}(t)$ ), we get

$$
\phi(t, s) \leqq \frac{K}{(t-s)^{\rho}} e^{c(t-s)}+K \int_{s}^{t} e^{c(t-\tau)} \phi(\tau, s) d \tau
$$

from which (6.5) with $\lambda=\rho$ follows.

Noting that $W(t, s)$ is uniformly bounded if $t-s \leqq 1$ (this follows from the proof of Theorem 5), and using (6.8) and (6.5) (with $\lambda=\rho$ ) for the case where $t-s>1$, we obtain (6.5) in the case $\lambda=0$. Finally, if $0<\lambda<\rho$, we use the inequality (see [15])

$$
\left\|A^{\lambda}(t) x\right\| \leqq K\left\|A^{\rho}(t) x\right\|^{\lambda / \rho}\|x\|^{1-\lambda / \rho} \quad\left(x \in D\left(A^{\rho}\right)\right)
$$

with $x=W(t, s) y$ and $y$ varying in $X$ and thus obtain (6.5).

The above proof can be modified to prove, under suitable assumptions, that $\left\|A^{\lambda}(t) W(t, s)\right\|$ decays exponentially. We shall need the following conditions on $V(t, s)$ and on $h$ :

$$
\begin{gathered}
\left\|A^{o}(t) V(t, s)\right\| \leqq K(t-s)^{-\rho} e^{-a(t-s)} \quad(a>0), \\
|h(t)|+|\ddot{h}(t)| \leqq \varepsilon e^{-b t} \quad(\varepsilon>0, b>0) .
\end{gathered}
$$

Corollary. Let the conditions $(\mathrm{A}),\left(\mathrm{B}_{\rho}\right),\left(\mathrm{H}^{\prime}\right)$ hold with $T=\infty$ and assume also that (6.9), (6.10) hold. If $\varepsilon$ is sufficiently small, depending on all the other constants which appear in all the previous assumptions, then there exists a positive number $\delta$ such that, for $0 \leqq s<t<\infty$,

$$
\left\|A^{\lambda}(t) W(t, s)\right\| \leqq K(t-s)^{-\lambda} e^{-\delta(t-s)} \quad \text { for any } 0 \leqq \lambda \leqq \rho .
$$

The proof is omitted.

7. Differentiability of solutions. Let $u(t)$ be a solution of (1.4). Then it satisfies (3.1). If $\dot{k}, h$ are uniformly Hölder continuous in closed subsets of $[0, T)$, then the same is true of the right-hand side of (3.1). Hence we can apply the differentiability theorem stated in $\$ 1.2$ for $m=0$, and conclude that $d u / d t$ and $A u$ are uniformly Hölder continuous in closed subsets of $(0, T)$.

Similarly, if $k, h$ have one strong derivative, uniformly Hölder continuous in closed subsets of $[0, T)$, then using the previous result we see that the same is true of the right-hand side of (3.1). Hence, if $A(t) A^{-1}(0)$ has one strong derivative which is uniformly Hölder continuous in closed subsets of $(0, T)$ then by the abovementioned differentiability theorem with $m=1$, it follows that $d^{2} u / d t^{2}$ and $A(d u / d t)$ exist and are uniformly Hölder continuous in closed subsets of $(0, T)$.

We can now differentiate (3.1) once with respect to $t$ and repeat the previous argument. Proceeding in this manner step-by-step, we arrive at the following theorem.

Theorem 7. Let (A), (B) hold and let $h(0)>0$. Assume that $A(t) A^{-1}(0)$ has $m$ strong derivatives $(m \geqq 0)$ which are uniformly Hölder continuous in closed subsets of 
$(0, T)$, and that $h(t), h(t)$ have $m+1$ (strong) derivatives which are Hölder continuous in closed subsets of $[0, T)$. Then any solution $u(t)$ of $(1.4)$ has $m+1$ strong derivatives, and these derivatives are uniformly Hölder continuous in closed subsets of $(0, T)$.

One can prove, by a method similar to [15], the following:

Theorem 8. Let (A), (B) hold and let $h(0)>0$. Assume that $A(t) A^{-1}(0)$ is analytic in $t$ in the interval $(0, T)$ and that $h(t), k(t)$ are also analytic in $t$ in the interval $[0, T)$. Then any solution $u(t)$ of $(1.4)$ is analytic in $t$ in the interval $(0, T)$.

REMARK. Differentiability of solutions for $t$ in the semiclosed interval $0 \leqq t<T$ can be obtained on the basis of Theorem 7. Suppose for simplicity that $k(0)=0$ and set $k(t) \equiv 0$ for $-1 \leqq t<0$. Extend $h(t), A(t)$ to $-1 \leqq t<0$ so that the extended functions are as smooth in $[-1, T)$ as the original functions in $[0, T)$. We can now rewrite (1.4) in the form

$$
u(t)=k(t)-\int_{-1}^{t} h(t-\tau) A(\tau) u(\tau) d \tau
$$

since, by uniqueness, $u(\tau) \equiv 0$ for $-1 \leqq \tau \leqq 0$. Now one can apply Theorem 7 in the interval $(-1, T)$ and thereby derive differentiability properties for $u(t)$ in $[0, T)$.

8. Other types of $A(t)$. In this section we generalize the results of $\$ \$ 1,5$ to equations with $A(t)$ satisfying conditions different from (A), (B); $A(t)$ is still a densely defined, closed linear operator with domain $D(A)$ independent of $t$. We impose the following conditions:

(A*) The resolvent of $A(t)$ exists for all $0 \leqq t \leqq T, \lambda \leqq 0$, and

$$
\left\|(\lambda I-A(t))^{-1}\right\| \leqq 1 /(c+|\lambda|) \text { for some } c>0 .
$$

(B*) $A(t) A^{-1}(0)$ is a bounded operator, strongly continuously differentiable in $[0, T]$.

It follows that for $s, t$ in $[0, T]$,

$$
\left\|A(t) A^{-1}(s)\right\| \leqq C, \quad\left\|A(s) A^{-1}(t)\right\| \leqq C, \quad\left\|A(t) A^{-1}(s)\right\| \leqq C
$$

where $\dot{A}(t) A^{-1}(s)$ is the strong derivative of $A(t) A^{-1}(s)$.

REMARK. The results stated below remain valid if $c=0$. Indeed, by a transformation of the form $u \rightarrow u e^{\varepsilon t}$ we may pass from the case $c=0$ to the case $c>0$.

Under the above conditions it was proved by Kato [9], [10] that the system

$$
\begin{aligned}
d u / d t+A(t) u & =f(t), \\
u(0) & =u_{0},
\end{aligned}
$$

has a unique solution provided $u_{0} \in D(A)$ and $f(t)$ is continuously differentiable. Furthermore, the solution can be represented in the form

$$
u(t)=U(t, 0) u_{0}+\int_{0}^{t} U(t, s) f(s) d s
$$


where $U(t, s)$ (the "fundamental solution") has the following properties:

$U(t, s)$ is strongly continuous in $(s, t)$ for $0 \leqq s \leqq t \leqq T ;$

$U(t, s) A^{-1}(s)$ is strongly continuously differentiable in $t$ for $s \leqq t \leqq T$;

$$
A(t) U(t, s) A^{-1}(s)
$$

is well defined and is strongly continuous in $t$ for $s \leqq t \leqq T$, and

$$
\frac{\partial}{\partial t} U(t, s) A^{-1}(s)=A(t) U(t, s) A^{-1}(s) .
$$

For later references we state the following lemma which is obtained from the proofs in [9], [10]:

LEMMA 8. Consider the integral

$$
F(t)=\int_{T_{0}}^{t} U(t, s) f(s) d s,
$$

where $f(t)$ is continuously differentiable in $\left[T_{0}, T\right]$ and $T_{0}$ is any point in $[0, T)$. Then $F(t) \in D(A)$ for any $t \in\left[T_{0}, T\right)$, and

$$
\|A(t) F(t)\| \leqq C\left[\sup _{T_{0} \leqq s \leqq t}\|f(s)\|+\sup _{T_{0} \leqq s \leqq t}\left\|\frac{d f(s)}{d t}\right\|\right]
$$

where $C$ is a constant independent of $T_{0}, f$.

We assume the following conditions on $h$ and $k$ :

$$
h(0)>0 \text { and } h \in C^{3}[0, T) .
$$

$\left(\mathrm{K}^{*}\right) \quad k(0) \in D(A)$ and $k(t)$ is twice continuously differentiable in $[0, T)$.

We can now proceed without difficulty to prove an analog of Theorem 1. For simplicity we assume that $h(0)=0$. Consider first the integral equation (3.10). To prove existence and uniqueness of a solution, we introduce the space $B_{0}^{*}$ of functions $v(t)$ from $\left[0, t_{0}\right]$ into $D(A)$ such that $v(t)$ and $A(t) v(t)$ are continuous in $\left[0, t_{0}\right]$, and the norm is

$$
\|v\|=\sup _{0<t<t_{0}}\|v(t)\|+\sup _{0<t<t_{0}}\|A(t) v(t)\| .
$$

We then notice, using $\left(\mathrm{B}^{*}\right)$, and $\left(\mathrm{H}^{*}\right)$, that $f(\tilde{u} ; t)$ (defined in (3.4)) is continuously differentiable. Hence the transformation $S v$ can be defined in the same way as in $\S 3$, and it maps $B_{0}^{*}$ into itself.

Instead of (3.17) we now have

$$
\left\|\frac{d}{d t} f(\tilde{u} ; t)\right\| \leqq \text { const } t\|u\| .
$$


Using Lemma 8 (instead of Lemma 1) we can then prove that $S$ maps $B_{0 M}^{*}$ into itself and is a contraction. Next, a step-by-step procedure yields the existence and uniqueness of a solution of $(3.10)$ for $0 \leqq t<T$. Since $f(\tilde{u} ; t)$ is continuously differentiable in $[0, T)$, by the previously stated results concerning (8.3) it follows that $u(t)$ is a solution of (1.4). Since conversely for every solution $u(t)$ of (1.4), the corresponding $\tilde{u}$ is a solution of (3.10) (here again we use the observation that $f(\tilde{u} ; t)$ is continuously differentiable), it follows that the above solution $u(t)$ is unique.

We have thus proved:

THEOREM 9. Assume that $\left(\mathrm{A}^{*}\right),\left(\mathrm{B}^{*}\right),\left(\mathrm{H}^{*}\right),\left(\mathrm{K}^{*}\right)$ hold. Then there exists a unique solution $u(t)$ of (1.4). $A(t) u(t)$ and $d u(t) / d t$ are continuous for $0 \leqq t<T$.

Theorem 2 extends to the present case only if we take $\mu=1$. Instead of (1.7) we now have: $A(t) W_{1}(t, s)$ is continuous in $t$ for $s \leqq t<T$. Note that $W_{1}(t, s)$ reduces to $U(t, s) A^{-1}(s)$ if $h \equiv 1$.

We define $W(t, s) \equiv W_{1}(t, s) A(s)$ as the fundamental solution of (1.4) or (1.5). (1.8) holds if $x \in D(A)$. (1.9) holds if $k(0) \in D(A), \dot{k}(s) \in D(A)$, and if $A(s) \dot{k}(s)$ is continuous in $[0, T)$.

The results of $\$ 5$ can be generalized for $0<\rho<1$. If $\rho=0$ and (for simplicity) $A$ is independent of $t$, then we can actually replace the condition $\left(\mathrm{A}^{*}\right)$ by the following weaker condition:

(A**) The resolvent of $A$ exists for all $\lambda \leqq 0$ and

$$
\left\|(\lambda I-A)^{-n}\right\| \leqq \frac{M}{(|\lambda|+c)^{n}} \quad(\lambda \leqq 0 ; n=1,2, \ldots)
$$

where $M, c$ are positive constants.

We mention that the condition $\left(\mathrm{A}^{* *}\right)$ with $c=0$ is necessary and sufficient in order for $A$ to generate a strongly continuous semigroup of bounded operators $e^{-t A}$ for $0 \leqq t<\infty$. Similarly, the condition ( $\left.\mathrm{A}^{*}\right)$ with $c=0$ and (A) are necessary and sufficient for $A$ to generate a strongly continuous semigroup of contraction operators and analytic semigroups respectively; for proofs, see Hille-Phillips [8] and Yosida [20].

In case $\left(\mathrm{A}^{* *}\right)$ holds, the previous assertions concerning (8.3) remain true (Phillips [13]). Hence, Theorem 9 remains true if the conditions $\left(\mathrm{A}^{*}\right),\left(\mathrm{B}^{*}\right)$ are replaced by $\left(\mathrm{A}^{* *}\right)$.

The results mentioned in the two paragraphs following Theorem 9 also remain true in the present case.

Proceeding analogously to $\S 5$ (with $\rho=0$ ), we find that if $\left(\mathrm{A}^{* *}\right)$ holds then $W(t, s)$ is a bounded continuous operator for $0 \leqq s \leqq t<T$. Furthermore,

$$
W(t, s)-e^{-(t-s) h(0) A}
$$

is strongly continuously differentiable in $t$, for $0 \leqq s \leqq t<T$. 
9. The case $h(0)=0$. We first assume that $h(0)=0$ but $h(0) \neq 0$.

Differentiating (1.4) twice with respect to $t$ and setting

$$
\tilde{u}(t)=u(t)+\frac{1}{h(0)} \int_{0}^{t} \ddot{h}(t-\tau) u(\tau) d \tau,
$$

we get

$$
d^{2} \tilde{u} / d t^{2}+h(0) A(t) \tilde{u}=f_{1}(\tilde{u} ; t)
$$

where

$$
\begin{aligned}
f_{1}(\tilde{u} ; t)= & \ddot{k}(t)+\int_{0}^{t} \ddot{h}(t-\tau)\left\{[A(t)-A(\tau)] A^{-1}(\tau)\right\} A(\tau) u(\tau) d \tau \\
& -\frac{\ddot{h}(0)}{h(0)} \frac{d u}{d t}-\frac{h^{(3)}(0)}{h(0)} u(t)-\frac{1}{h(0)} \int_{0}^{t} h^{(4)}(t-\tau) u(\tau) d \tau .
\end{aligned}
$$

Substituting $\tilde{u}=\hat{u} e^{s t}$ where $2 \varepsilon=-\tilde{h}(0) / h(0), \hat{A}(t)=h(0) A(t)+\delta$ where $\delta$ is an appropriate constant, (9.2) takes the form

$$
d^{2} \hat{u} \mid d t^{2}+\hat{A}(t) u=\hat{f}_{1}(\hat{u} ; t)
$$

where

$$
\begin{gathered}
\hat{f}_{1}(\hat{u} ; t)=e^{-\delta t} \tilde{k}(t)+\int_{0}^{t} e^{-\delta(t-\tau)} \hat{h}(t-\tau)\left\{[A(t)-A(\tau)] A^{-1}(\tau)\right\} \\
A(\tau)\left[e^{-\varepsilon \tau} u(\tau)\right] d \tau+\int_{0}^{t} g(t-\tau) \hat{u}(\tau) d \tau
\end{gathered}
$$

where $g(t)$ is a suitable function which belongs to the same differentiability class as $h^{(4)}(t)$.

Formally, (1.4) is equivalent to the equation (9.4) with the initial conditions

$$
\begin{aligned}
\hat{u}(0) & =k(0), \\
d \hat{u}(0) / d t & =\dot{k}(0)+(\vec{h}(0) / 2 h(0)) k(0) .
\end{aligned}
$$

One can now apply known results for $(9.4),(9.6)$ in case $\hat{f}_{1}(\tilde{u} ; t)$ is replaced by a given function of $t$ in order to solve the system (9.4), (9.6). Thus, in some instances, if $A(t)$ is as in $\S 1$ and if $h(0)>0, \delta \geqq 0$ then there is a way of reducing (9.4), (9.6) to a system of the form

$$
\begin{aligned}
\frac{d v}{d t}+B(t) v & =f^{*}(v ; t), \\
v(0) & =v_{0},
\end{aligned}
$$

where $B(t)$ is as in $\S 8$ and $f^{*}(v ; t)$ has a form similar to $(9.5)$. The results of $\S 8$ can then be applied, thus yielding the existence of a unique solution of (1.4).

EXAMPLE. $A(t)=-\Delta$ where $\Delta$ is the Laplace operator in 3 variables $(D(A)$ can be defined in various ways). (9.4) has the form

$$
\partial^{2} \hat{u} / \partial t^{2}-\hat{\Delta} \hat{u}=\hat{f}_{1}(\hat{u} ; t)^{\bullet}(\hat{\Delta}=h(0) \Delta-\delta) .
$$


The reduction to the form (9.7) with $B$ as in $\S 8$ is done by setting $v=(u, w)$ where $w=\left(w_{1}, w_{2}, w_{3}\right)$ is given by

$$
\operatorname{div} w=\frac{\partial u}{\partial t}, \quad \frac{\partial w}{\partial t}=\operatorname{grad} u
$$

For the Cauchy problem corresponding to the same operator $A(t)=-\Delta$, the fundamental solution (of the wave operator) is known explicitly and one can therefore treat (9.8), (9.6) directly (instead of reducing it to the form (9.7)).

Consider now the case where $h^{(j)}(0)=0$ for $j=0,1, \ldots, m-1$, and $h^{(m)}(0) \neq 0$. By differentiating (1.4) $m+1$ times, we obtain an equation of the form

$$
\frac{d^{m+1} \tilde{u}}{d t^{m+1}}+\sum_{j=0}^{m} \alpha_{j} \frac{d^{m-j} \tilde{u}}{d t^{m-j}}+h^{(m)}(0) A(t) \tilde{u}=f_{m}(\tilde{u} ; t) \quad\left(\alpha_{j} \text { constants }\right) .
$$

If one can solve the corresponding Cauchy problem with $f_{m}(\tilde{u} ; t)$ replaced by a given function of $t$, and if one can further establish suitable a priori estimates on the solution (or estimates on the fundamental solution), then one may also be able to solve the Cauchy problem for (9.9) using a fixed-point theorem.

10. Applications and generalizations. Let $x=\left(x_{1}, \ldots, x_{n}\right)$ be a variable point in the $n$-dimensional euclidean space $R^{n}$, and let $B \subset R^{n}$ be a bounded domain with a smooth boundary $\partial B$. Set $\Omega=B \times(0, T)$. We consider linear partial differential operators

$$
L(x, t, D)=\sum_{|\alpha| \leqq \nu} a_{\alpha}(x, t) D^{\alpha}
$$

with smooth coefficients $a_{\alpha}(x, t)$ in $\bar{\Omega}$, where $\alpha=\left(\alpha_{1}, \ldots, \alpha_{n}\right),|\alpha|=\alpha_{1}+\cdots+\alpha_{n}$, $D^{\alpha}=\partial^{|\alpha|} / \partial x_{1}^{\alpha} \ldots \partial x_{n}^{\alpha}{ }_{n} . L$ is said to be strongly elliptic if $\nu=2 m$ and

$$
(-1)^{m} \operatorname{Re}\left\{\sum_{|\alpha|=2 m} a_{\alpha}(x, t) \xi^{\alpha}\right\} \geqq \gamma|\xi|^{2 m} \quad(\gamma>0)
$$

for all $(x, t) \in \Omega$, where $\xi^{\alpha}=\dot{\xi}_{1}^{\alpha} \ldots \xi_{n}^{\alpha^{n}}$ and $\gamma$ is a constant independent of $(x, t)$.

We take $X$ to be the Banach space $W_{p}^{2 m}(B)(1<p<\infty)$ consisting of all functions in $L^{p}(B)$ whose first $2 m$-derivatives in the sense of distributions are also functions in $L^{p}(B)$. The norm is given by

$$
\|u\|=\sum_{|\alpha| \leqq 2 m}\left\|D^{\alpha} u\right\|_{L^{p}(B)}
$$

Denote by $A_{0}(t)$ the operator $L(x, t, D)$ defined on all the $C^{\infty}$ functions in $B$ which satisfy homogeneous "regular" elliptic boundary conditions on $\partial B$ which are independent of $t$. The simplest example of such conditions is provided by the Dirichlet conditions, i.e., the conditions that the function and all its normal derivatives up to order $m-1$ are zero on $\partial B$.

The operator $A_{0}(t)$ can be extended to a closed operator $\bar{A}_{0}(t)$. It is known (see Agmon [1] and the references given there) that there exists a number $\gamma_{0}$ such that 
the operator $A(t)=\bar{A}_{0}(t)+\gamma_{0} I$ has a domain dense in $X$ and independent of $t$, and satisfies the conditions (A), (B) of $\S 1$.

Applying Theorems 1, 7 and the Sobolev inequalities, we conclude that there exists a unique solution $u(x, t)$ of (1.4) and it is sufficiently smooth in $\bar{B} \times(\varepsilon, T)$ for any $\varepsilon>0$.

As for the condition $\left(\mathrm{B}_{\rho}\right)$, it follows from results of Kato [11] that if $X=W_{2}^{2 m}(B)$ then, for some class of elliptic boundary conditions (the Dirichlet conditions are included), the domain of $A^{\rho}(t)$ is independent of $t$ if $0 \leqq \rho<1 / 2$. From [7] it also follows that (5.1) holds (when $X=W_{2}^{2 m}(B)$ ) if all the coefficients $a_{\alpha}(x, t)$, in (8.1), with $|\alpha| \geqq 2 m \rho-n / 2$ are independent of $t$.

The results of $\S 8$ apply to "hyperbolic" equations. As an example we consider (1.4) with $A(t)$ the operator $-i L(x, t, D) u$ arising in the Schrödinger equation, i.e., $L$ is a second-order elliptic operator with smooth coefficients in $\bar{\Omega}=\bar{B} \times[0, T]$. With a standard boundary condition, the conditions $\left(\mathrm{A}^{*}\right),\left(\mathrm{B}^{*}\right)$ are satisfied if the coefficients of $L$ and the boundary conditions are sufficiently smooth.

Generalizations. Most of the results of the present chapter extend with minor modifications to integral equations of the form

$$
u(t)=k(t)-\int_{0}^{t} h(t-\tau, \tau) A(\tau) u(\tau) d \tau
$$

(the condition $h(0)>0$ is replaced by $h(0, t)>0)$. This is true also if $h(t-\tau, \tau)$ is not a scalar function but a bounded operator which commutes with the $A(s)$, provided the operators $A^{*}(t) \equiv h(0, t) A(t)$ satisfy the same conditions that the $A(t)$ were assumed to satisfy in the previous sections.

We finally mention that Theorem 1 can be extended to nonlinear integral equations of the form

$$
u(t)=k(t)-\int_{0}^{t} h(t-\tau) A(\tau) u(\tau) d \tau+\int_{0}^{t} g(t, \tau, u(\tau)) d \tau
$$

provided that for any $R>0$, the conditions $\|v\| \leqq R,\|w\| \leqq R$ imply

$$
\left\|g\left(t, \tau, A^{-\rho}(0) v\right)-g\left(t^{\prime}, \tau, A^{-\rho}(0) w\right)\right\| \leqq C(R)\left(\left|t-t^{\prime}\right|^{v}+\|v-w\|\right)
$$

for some $\nu \in(0,1]$. However, the existence and uniqueness theorem we get is only a local one. The proof is obtained by combining the proof of Theorem 1 with the proof of Theorem 7 in [15]. The case where $A=A(t, u)$ is nonlinear in $u$ can also be treated in this way.

11. Some results in Hilbert space. Let $E$ be a Hilbert space, and let $A$ be a selfadjoint, positive operator in $A$. Suppose $u$ is a solution of

$$
\begin{aligned}
d u / d t+A u & =f(t) \quad(0<t<T), \\
u(0) & =u_{0}
\end{aligned}
$$


where $u_{0} \in D\left(A^{1 / 2}\right)$ and $f(t)$ is continuous in [0,T). We then have (cf. [14]):

LEMMA 9. Under the foregoing assumptions,

$$
\int_{0}^{s}\|A u\|^{2} d t \leqq \int_{0}^{s}\|f\|^{2} d t+\left\|A^{1 / 2} u_{0}\right\|^{2} \quad \text { for } 0<s<T .
$$

Proof. It suffices to consider the case where $u$ is sufficiently smooth, since the general case then follows by approximation. We have:

$$
\|f\|^{2}=\|d u / d t+A u\|^{2}=\|d u / d t\|^{2}+\|A u\|^{2}+J
$$

where

$$
J=(d u / d t, A u)+(A u, d u / d t)=d(u, A u) / d t=d\left\|A^{1 / 2} u\right\|^{2} / d t .
$$

Now integrate (11.4) and use the last expression for $J$.

Lemma 9 can be used to solve (1.4) in case $u, k$ are functions with values in the Hilbert space $E$ and $A(t)$ is "nearly self-adjoint." We maintain the assumption (A), but replace (B) by the following assumption:

(C) (i) For every $s \in[0, T)$ there exists a self-adjoint positive operator $A_{s}$ with domain equal to $D(A)$, such that, for any $\varepsilon>\varepsilon_{s}(t-s)$,

$$
\left\|A_{s} u-A(t) u\right\| \leqq \varepsilon\left\|A_{s} u\right\|+C_{s}(\varepsilon)\|u\| ;
$$

here $\varepsilon_{s}(t)$ is a continuous function with $\varepsilon_{s}(t)>0$ if $t \neq 0, \varepsilon_{s}(0)=0$ and $C_{s}(\varepsilon)$ is a constant satisfying: $C_{s}(\varepsilon) \rightarrow \infty$ if $\varepsilon \rightarrow 0$.

(ii) $A_{s} A^{-1}(t)$ and $A(t) A_{s}^{-1}$ are continuous operators in $t$.

Under the conditions $(\mathrm{A}),(\mathrm{C}),(\mathrm{K})$ we can then prove an existence and uniqueness theorem for (1.4) provided only $h(0)>0$ and $h \in C^{1}[0, T)$.

The proof is obtained step-by-step on $t$-intervals. In the first step we consider (3.1) and rewrite it in the form

$$
\frac{d u}{d t}+h(0) A_{0} u=h(0)\left[A_{0}-A(t)\right] u(t)+\dot{k}(t)-\int_{0}^{t} h(t-\tau) A(\tau) u(\tau) d \tau .
$$

We then apply the technique of $\S 3$, employing Lemma 9 instead of Lemma 1. Further details are omitted.

It is worth noting that the condition $(\mathrm{C})$ is satisfied for strongly elliptic equations (with "regular" elliptic boundary conditions) when the leading coefficients are realvalued (compare §7).

Using the equation (3.1) one can immediately extend the differentiability and analyticity theorems of Friedman [6] (which were derived for equations of the form (2.3)) to solutions of (1.4). Using the notation of [6], the differentiability theorem takes the following form:

If $k(t) \in H^{m}(0, T), h(t) \in C^{m}[0, T)$ and $A(t) A^{-1}(s)$ has $m-1$ continuous $t$ derivatives, where $m \geqq 2$, and if, finally, (1.1) holds for all $\lambda$ pure imaginary with 
$|\lambda| \geqq N$ where $C$ and $N$ may depend on $t$, then any solution of $(1.4)$ is in $H^{m}(\varepsilon, T-\varepsilon)$ for any $\varepsilon>0$.

The analyticity result is similar.

We conclude with a uniqueness theorem. We shall assume that (1.1) holds for $\operatorname{Re} \lambda=\sigma_{n}$ where $\left\{\sigma_{n}\right\}$ is a sequence of negative numbers which converges to $-\infty$, and (instead of (1.2)) that

$$
\left\|[A(t)-A(\tau)] A^{-1}(\tau)\right\| \leqq \delta(t-\tau)
$$

where $\delta(t)$ is a continuous function with $\delta(0)=0$. We finally assume that $h(0)>0$ and $h \in C^{1}[0, T)$. We then have:

Under the foregoing assumptions, if $u$ is a solution (1.4) with $k \equiv 0$ then $u \equiv 0$.

The proof is similar to the proof of Theorem 1 of [4].

\section{Chapter 2. Global Bounds and Asymptotic Behavior}

In this chapter we consider the case where $A(t)$ is independent of $t$, but we derive very precise global bounds on the fundamental solution. In fact, under suitable conditions on $h$ and $A$, we prove that some of these bounds are both necessary and sufficient in order that a certain relation between $h$ and $A$ should hold. The bounds are then used to study the asymptotic behavior of solutions.

1. Preliminaries. Let $X$ be a complex Banach space. By $L^{p}(a, b ; X)$ (with $1 \leqq p \leqq \infty)$ we denote the Banach space consisting of equivalence classes of measurable functions $f(t)$ from $(a, b)$ into $X$ with finite norm

$$
\|f(t)\|_{L^{p}(a, b)} \equiv\left\{\int_{a}^{b}\|f(t)\|^{p} d t\right\}^{1 / p}
$$

When there can be no confusion about the interval $(a, b)$, we denote this norm simply by $\|f(t)\|_{L^{p}}$.

If $f(t)$ is a function from $(0, \infty)$ into $X$, its Laplace transform $f^{\wedge}(s)$ is defined by

$$
f^{\wedge}(s)=\int_{0}^{\infty} e^{-s t} f(t) d t
$$

whenever it exists.

We denote by $H^{p}(\alpha ; X)$ the class of all functions $g(s)$ from $\operatorname{Re} s>\alpha$ into $X$, which are analytic in $\operatorname{Re} s>\alpha$ and satisfy the following conditions:

(a) $\sup _{\sigma>\alpha}\left[\int_{-\infty}^{\infty}\|g(\sigma+i \tau)\|^{p} d \tau\right]^{1 / p}<\infty$;

(b) $g(\alpha+i \tau)=\lim _{\sigma \rightarrow \alpha} g(\sigma+i \tau)$ exists almost everywhere and belongs to $L^{p}(-\infty, \infty ; X)$.

We shall need the following result [8, Theorem 6.3.2]:

Lemma 1. Let $f(t) \in L^{1}(0, T ; X)$ for any $T>0$ and assume that $\int_{0}^{\infty} e^{-s t} f(t) d t$ is absolutely convergent for any $s$ with $\operatorname{Re} s>\alpha$ where $\alpha \geqq 0$. Set

$$
f(t, w)=\frac{1}{2 \pi} \int_{-w}^{w}\left(1-\frac{|\tau|}{w}\right) e^{(\gamma+i \tau) t} f^{\wedge}(\gamma+i \tau) d \tau \quad(\gamma>\alpha) .
$$


Then $\lim _{w \rightarrow \infty} f(t, w)$ exists and equals $f(t)$ for almost every $t>0$, and equals zero for $\boldsymbol{t}<\mathbf{0}$.

CoRollary. If $f_{1}, f_{2}$ satisfying the same assumptions as $f$, and if $f_{1}^{\wedge}(s) \equiv f_{2}^{\wedge}(s)$ for $\operatorname{Re} s>\alpha$, then $f_{1}(t)=f_{2}(t)$ for almost all $t>0$.

We shall also need

Lemma 2. Let $g(s) \in H^{1}(\alpha ; X), \alpha \geqq 0$. Then the function

$$
f(t)=\frac{1}{2 \pi i} \int_{\gamma-i \infty}^{\gamma+i \infty} e^{s t} g(s) d s \quad(\gamma \geqq \alpha)
$$

is defined and independent of $\gamma$ for all $t \in(-\infty, \infty) . f(t)$ is zero for $t<0$, is continuous in $t$, its Laplace transform coincides with $g(s)$, and if $\alpha=0$ it converges to 0 as $t \rightarrow \infty$.

The fact that $f$ is independent of $\gamma$ follows from [8, Theorem 6.4.1] and Cauchy's theorem; the other parts of the lemma follow from the remark following Theorem 6.6.1 of [8] and the usual proof of the Riemann-Lebesgue theorem.

We denote by $B(X)$ the Banach space of bounded linear operators on $X$. For any linear operator $A$ on $X$, we denote by $\sigma(A)$ and $\rho(A)$ the spectrum and the resolvent sets of $A$, respectively.

Definition. A measurable function $S(t)$ from $(0, \infty)$ into $B(X)$ is called a fundamental solution of the equation (1.1.4) if $S(t) \in L^{1}(0, T ; B(X))$ for any $T>0$ and if

$$
S(t)=I-A \int_{0}^{t} h(t-\tau) S(\tau) d \tau \text { for almost all } t>0 .
$$

We shall also call $S(t)$ a fundamental solution of (1.1).

As in Chapter 1, we cannot introduce $A$ inside the integral since $A S(\tau)$ is generally not integrable in $(0, T)$ for any $T>0$ (even if $h \equiv 1)$. $S(t)$ may be conceived as a "weak solution" of (1.5). A different type of weak solution was considered in subsection 1.5. It will be shown later on that if $h$ is sufficiently smooth then the fundamental solution $S$ (as presently defined) coincides with the fundamental solution $W$ of Chapter 1 .

We now introduce several conditions which will be used throughout this chapter.

Definition. A linear operator $A$ in $X$ is said to belong to the class $\mathfrak{A}$ if:

(i $\left.\mathrm{i}_{\mathrm{A}}\right) \quad A$ is closed and densely defined;

(ii $\mathrm{A}_{\mathrm{A}} \sigma(A) \subset\left\{\lambda ;|\arg \lambda| \leqq \pi / 2-\varepsilon, \operatorname{Re} \lambda \geqq \lambda_{0}\right\}$ for some $\varepsilon>0, \lambda_{0}>0$;

(iii $\left.{ }_{\mathrm{A}}\right)\left\|(\lambda I-A)^{-1}\right\| \leqq c /|\lambda|$ if $|\arg \lambda|>\pi / 2-\varepsilon$.

Note that, for fixed $t$, the condition (A) of $\S 1.1$ coincides with the conditions (ii $\left.{ }_{\mathrm{A}}\right),\left(\mathrm{iii}_{\mathrm{A}}\right)$.

Definition. A scalar function $h(t)$ is said to belong to the class $\mathfrak{S}$ if:

(i $\left.\mathrm{i}_{\mathrm{h}}\right) h(0)>0$ and $h(t)$ is absolutely continuous in $[0, T)$ for any $T>0$;

(ii $\mathrm{i}_{\mathrm{h}} h(t) \in L^{1}(0, \infty)$. 
Since $h \in L^{1}(0, \infty)$, we can introduce the function

$$
g(s)=h(0)+h^{\wedge}(s) \text { for } \operatorname{Re} s \geqq 0 .
$$

Since $h(t)$ is a bounded function, the right-hand side of (1.2) is equal to $s h^{\wedge}(s)$ if $\operatorname{Re} s>0$. Hence we can define $h^{\wedge}(s)$ by continuity to $\operatorname{Re} s \geqq 0, s \neq 0$ and thus obtain:

$$
g(s)=h(0)+h^{\wedge}(s)=s h^{\wedge}(s) \text { for } \operatorname{Re} s \geqq 0, s \neq 0 .
$$

If $g(0) \neq 0$, then, by continuity, $h^{\wedge}(0)=\infty$; thus $1 / h^{\wedge}(0)=0$. In general define $1 / h^{\wedge}(0)$ as the set of all limit points of $1 / h^{\wedge}(s)$ as $s \rightarrow 0, \operatorname{Re} s \geqq 0$.

The set

$$
\Delta \equiv\left\{-1 / h^{\wedge}(s) ; \operatorname{Re} s \geqq 0\right\}
$$

will play a fundamental role throughout this chapter.

Finally we remark that the complex plane will be denoted by $\mathbf{C}$.

2. $S$ in $L^{p}(0, \infty ; B(X))$ for $2 \leqq p<\infty$. The main result of the present section is the following:

THEOREM 1. Let $h \in \mathfrak{S}, A \in \mathfrak{A}$. If

$$
\Delta \subset \rho(A) \mid\{\infty\} \text { and } g(0) \neq 0
$$

then there exists a fundamental solution $S(t)$ of (1.1) and $S(t)$ belongs to $L^{p}(0, \infty ; B(X))$ for any $p$ with $2 \leqq p<\infty$. If $S_{0}(t)$ is another fundamental solution and if $e^{\alpha t} S_{0}(t)$ belongs to $L^{r}(0, \infty ; B(X))$ for some $r \geqq 1, \alpha \geqq 0$, then $S_{0}(t)=S(t)$ almost everywhere.

Note that the conditions in (2.1) can be stated equivalently as the following conditions:

$$
\begin{gathered}
\Delta \subset \rho(A), \\
g(s) \neq 0 \quad \text { for all } s \text { with } \operatorname{Re} s \geqq 0 .
\end{gathered}
$$

Lemma 3. Let $h \in \mathfrak{H}, A \in \mathfrak{A}$ and let (2.2) hold. Then there exists an open set $\Lambda$ in the complex plane having the following properties:

$$
\begin{gathered}
\sigma(A) \subset \Lambda, \\
\Delta \cap \bar{\Lambda}=\varnothing, \\
\bar{\Lambda} \cap\left\{\lambda ;|\lambda| \geqq \lambda_{1}\right\} \subset\{\lambda ;|\arg \lambda| \leqq \pi / 2-\varepsilon / 2\} \text { for some } \lambda_{1}>0, \\
\bar{\Lambda} \subset\left\{\lambda ; \operatorname{Re} \lambda \geqq \lambda_{2}\right\} \quad \text { for some } \lambda_{2}>0 .
\end{gathered}
$$

$\Lambda$ is a finite union of domains, of which all but one are bounded, and the boundary $C$ of $\Lambda$ consists of a finite number of smooth Jordan arcs. 
Proof. For any $s_{0}>0$, set

$$
\begin{aligned}
& \Delta_{1}\left(s_{0}\right)=\left\{-1 / h^{\wedge}(s) ; \operatorname{Re} s \geqq 0,|s| \geqq s_{0}\right\}, \\
& \Delta_{2}\left(s_{0}\right)=\left\{-1 / h^{\wedge}(s) ; \operatorname{Re} s \geqq 0,|s|<s_{0}\right\} .
\end{aligned}
$$

By (1.2),

$$
s h^{\wedge}(s)=h(0)+o(1) \text { as }|s| \rightarrow \infty .
$$

Hence,

$$
\left|\arg s+\arg h^{\wedge}(s)\right|<\varepsilon / 3
$$

if, say, $|s| \geqq s_{0}$. Since $\operatorname{Re} s \geqq 0$, this means that

$$
\left|\pi-\arg \left[-1 / h^{\wedge}(s)\right]\right|<\pi / 2+\varepsilon / 3 .
$$

Consequently,

$$
\Delta_{1}\left(s_{0}\right) \subset\{\lambda ;|\pi-\arg \lambda|<\pi / 2+\varepsilon / 3\} .
$$

From (2.4) it also follows that

$$
\left|-1 / h^{\wedge}(s)\right| \geqq s_{1} / 2 h(0) \text { if }|s| \geqq s_{1},
$$

provided $s_{1}$ is a sufficiently large number. Thus, if $s_{2}=\max \left(s_{0}, s_{1}\right)$,

$$
\Delta_{1}\left(s_{2}\right) \subset\left\{\lambda ;|\pi-\arg \lambda|<\pi / 2+\varepsilon / 3,|\lambda| \geqq \lambda_{1} \equiv s_{1} / 2 h(0)\right\} .
$$

The domain

$$
\Lambda_{1}=\left\{\lambda ;|\arg \lambda|<\pi / 2-\varepsilon / 2,|\lambda|>\lambda_{1}\right\}
$$

is going to be the part of $\Lambda$ which lies outside the circle $|\lambda|=\lambda_{1}$. It remains to define the part of $\Lambda$ which lies in the disk $|\lambda| \leqq \lambda_{1}$.

The sets $\Delta^{\prime}=\Delta \cap\left\{\lambda ;|\lambda| \leqq \lambda_{1}\right\}, \sigma^{\prime}=\sigma(A) \cap\left\{\lambda ;|\lambda| \leqq \lambda_{1}\right\}$ are disjoint compact sets, and the points $z_{j}=\lambda_{1} e^{i \theta_{j}}(j=1,2)$ where $\theta_{1}=\pi / 2-\varepsilon / 2, \theta_{2}=-\pi / 2+\varepsilon / 2$ do not belong to these sets. Furthermore, the arc $\Gamma$ on $|\lambda|=\lambda_{1}$ joining $z_{1}$ to $z_{2}$ clockwise does not intersect $\Delta^{\prime}$, whereas the complementary part $\Gamma^{*}$ of the boundary of

$$
\left\{\lambda ;|\lambda|<\lambda_{1}, \operatorname{Re} \lambda>0\right\}
$$

does not intersect $\sigma^{\prime}$.

Given a compact set $B$, its complement consists of domains, one of which is unbounded. Each of the bounded domains will be called a hole of $B$.

We claim that there are no holes of $\sigma^{\prime} \cup \Gamma$ which contain points of $\Delta^{\prime}$. Indeed, suppose there is a point $\lambda_{0} \in \Delta^{\prime}$ lying in a hole $H$ of $\sigma^{\prime} \cup \Gamma$. Observe, by (2.4), that $\Delta$ contains points $\lambda^{\prime}$ with $\left|\lambda^{\prime}\right|>\lambda_{1}$. Since $\Delta$ is a domain, there is an arc $\gamma$ connecting $\lambda_{0}$ to $\lambda^{\prime}$ and lying in $\Delta$. This arc must intersect $\sigma^{\prime} \cup \Gamma$, which is impossible since $\Delta \cap \Gamma=\varnothing$ (by (2.5)) and $\Delta \cap \sigma^{\prime}=\varnothing$ (by (2.2)).

Suppose first that there are also no holes of $\Delta^{\prime} \cup \Gamma^{*}$ which contain points of $\sigma^{\prime}$, and denote by $\Delta^{*}, \sigma^{*}$ the sets obtained by "filling" the holes of $\Delta, \sigma$ respectively. 
Then $\Delta^{*} \cap \sigma^{*}=\varnothing$. It is easy to show that there is a smooth arc $C_{2}$ joining $z_{1}$ to $z_{2}$ which does not intersect $\Delta^{*} \cup \sigma^{*}$ and such that the union of $\Gamma$ and the interior $\Lambda_{2}$ of $C_{2} \cup \Gamma$ contains $\sigma^{*}$, but $\bar{\Lambda}_{2} \cap \Delta^{*}=Q$. (One way of showing this is to introduce a mesh by lines parallel to the coordinate axes, replace $\Delta^{*}, \sigma^{*}$ by larger closed sets $\tilde{\Delta}$, $\tilde{\sigma}$ with $\tilde{\Delta} \cap \tilde{\sigma}=Q$ such that each consists of a finite number of closed polygons bounded by lines of the mesh.) Thus, the lemma is proved if there are no holes of $\Delta^{\prime} \cup \Gamma^{*}$ containing points of $\sigma^{\prime}$.

It remains to consider the case where some holes of $\Delta^{\prime} \cup \Gamma^{*}$ do contain points of $\sigma(A)$. Enumerate these holes and denote them by $H_{j}$. If their number is finite, then we modify the previous argument, filling all the holes other than the $H_{j}$. We then construct $C_{2}$ as before such that the union of $\Gamma$ and the interior of $C_{2} \cup \Gamma$ contains all the points of $\sigma^{\prime}$ except those which lie in the $H_{j}$. We next construct in each $H_{j}$ a domain $P_{j}$ containing $\sigma^{\prime} \cap H_{j}$, such that $\bar{P}_{j} \cap \Delta=Q$. Now we take $\Lambda_{2}$ as the union of the $P_{j}$ and the interior of $C_{2} \cup \Gamma$. It is easily verified that $\Lambda=\operatorname{int}\left(\bar{\Lambda}_{1} \cup \bar{\Lambda}_{2}\right)$ has all the required properties.

To complete the proof of the lemma, we show that the number of the $H_{j}$ must always be finite. Indeed, if not, then the area of $H_{j}$ tends to 0 as $j \rightarrow \infty$. We can therefore pick, for each $j$, one point $\alpha_{j}$ in $H_{j} \cap \sigma^{\prime}$ and another point $\beta_{j}$ in $\Delta^{\prime}$ such that $\left|\alpha_{j}-\beta_{j}\right| \rightarrow 0$ as $j \rightarrow \infty$. $\beta_{j}$ can be written in the form

$$
\beta_{j}=-1 / h^{\wedge}\left(\sigma_{j}\right) \text { for some } \sigma_{j} \text { with }\left|\sigma_{j}\right| \leqq s_{2}, \operatorname{Re} \sigma_{j} \geqq 0 \text {. }
$$

We may assume that $\sigma_{j} \rightarrow \sigma^{*}$. Then

$$
\alpha \equiv \lim \alpha_{j}=\lim \beta_{j}=-1 / h^{\wedge}\left(\sigma^{*}\right) .
$$

Since the right-hand side lies in $\Delta$ whereas the left-hand side lies in $\sigma(A)$ (since $\sigma(A)$ is a closed set), we get a contradiction to (2.2).

Lemma 4. Let $h \in \mathfrak{F}, A \in \mathfrak{A}$ and let (2.1) hold. Let $\Lambda$ be as in Lemma 3. Then there exists a positive constant $c$ such that

$$
\left|h^{\wedge}(s) /\left(1+\lambda h^{\wedge}(s)\right)\right| \leqq c /(|\lambda|+|s|)
$$

for all $\lambda \in \bar{\Lambda}$ and for all $s$ with $\operatorname{Re} s \geqq 0$.

Notation. In this and in the following sections, various positive constants independent of $\lambda$ and $s$ will be denoted by $c$.

Proof. Rewrite (2.6) in the form

$$
\left|1 / h^{\wedge}(s)+\lambda\right|>c(|\lambda|+|s|) .
$$

If (2.7) is not satisfied then there exist sequences $\left\{s_{n}\right\},\left\{\lambda_{n}\right\}$ with $\operatorname{Re} s_{n} \geqq 0, \lambda_{n} \in \bar{\Lambda}$ such that

$$
\left|1 / h^{\wedge}\left(s_{n}\right)+\lambda_{n}\right| \leqq\left(\left|\lambda_{n}\right|+\left|s_{n}\right|\right) / n .
$$

We may assume that each of these sequences is either convergent or divergent to $\infty$. 
If $s_{n} \rightarrow s^{*}$, there are two possibilities: either $\lambda_{n} \rightarrow \lambda^{*}$, in which case (2.8) implies

$$
1 / h^{\wedge}\left(s^{*}\right)+\lambda^{*}=0
$$

contradicting the fact that $\Delta \cap \bar{\Lambda}=\varnothing$, or $\lambda_{n} \rightarrow \infty$, in which case (2.8) becomes: $\left|\lambda_{n}\right| \leqq\left|\lambda_{n}\right| / n+c$, which is impossible.

Suppose next that $s_{n} \rightarrow \infty$. Using (2.4), we obtain from (2.8)

$$
\left|s_{n} / h(0)+o\left(s_{n}\right)+\lambda_{n}\right| \leqq\left(\left|\lambda_{n}\right|+\left|s_{n}\right|\right) / n .
$$

If $\lambda_{n} \rightarrow \lambda^{*}$ then it follows that $\left|s_{n}\right| \leqq c\left|s_{n}\right| / n+c$, which is impossible. Hence $\lambda_{n} \rightarrow \infty$.

Consider next the case where $\left\{s_{n} / \lambda_{n}\right\}$ is a convergent sequence. By selecting a subsequence we may assume that $\lambda_{n} /\left|\lambda_{n}\right| \rightarrow e^{i \theta}$. Since $\lambda_{n} \in \bar{\Lambda}$, we have $\cos \theta>0$. Dividing both sides of (2.9) by $\left|\lambda_{n}\right|$ and letting $n \rightarrow \infty$, we get

$$
\left|\lim \left(s_{n} /\left|\lambda_{n}\right|\right)+\cos \theta+i \sin \theta\right|=0 .
$$

Since $\operatorname{Re} s_{n} \geqq 0$, the last equality is impossible.

Thus we may assume that $s_{n} / \lambda_{n} \rightarrow \infty$, i.e., $\lambda_{n} / s_{n} \rightarrow 0$. Dividing both sides of (2.9) by $\left|s_{n}\right|$ and letting $n \rightarrow \infty$, we then get $1 / h(0)=0$, which is impossible. This completes the proof of the lemma.

We proceed to the construction of a fundamental solution. First we obtain explicit formulas by formal calculations. Taking the Laplace transform of (1.1) we get $S^{\wedge}(s)=I / s-A h^{\wedge}(s) S^{\wedge}(s)$, so that

$$
S^{\wedge}(s)=[s I+g(s) A]^{-1} .
$$

The right-hand side can be expressed by the Dunford integral (see [2, p. 601])

$$
\frac{1}{2 \pi i} \int_{C}(\lambda I-A)^{-1} \hat{S}_{\lambda}(s) d \lambda
$$

where

$$
\hat{S}_{\lambda}(s)=1 /(s+\lambda g(s)) \quad(\lambda \in \bar{\Lambda}, \operatorname{Re} s \geqq 0)
$$

and $C$ is as in Lemma 3. Thus we shall first study the function in (2.12). (But see the remark at the end of this section.)

Lemma 5. Let $h \in \mathfrak{H}, A \in \mathfrak{A}$ and let (2.1) hold. Then the function $\hat{S}_{\lambda}(s)$ is analytic for $\operatorname{Re} s>0$, continuous for $\operatorname{Re} s \geqq 0$, and satisfies

$$
\left|\hat{S}_{\lambda}(s)\right| \leqq c /(|\lambda|+|s|)
$$

Proof. The assertions concerning analyticity and continuity follow from the corresponding properties for $g(s)$ and the fact (see Lemma 3) that $s+\lambda g(s) \neq 0$ if $\lambda \in \bar{\Lambda}, \operatorname{Re} s \geqq 0$. Next,

$$
\left|\hat{S}_{\lambda}(s)\right|=1 /\left|s\left[1+\lambda h^{\wedge}(s)\right]\right| \leqq c /|g(s)|(|\lambda|+|s|)
$$


by Lemma 4 . Since, by (1.2) and (2.3),

$$
|1 / g(s)| \leqq c,
$$

(2.13) follows.

From Lemma 5 it follows that $S_{\lambda}(s)$ belongs to $H^{p}(0 ; \mathbf{C})$, where $p$ is any number with $1<p \leqq \infty$. Therefore, a standard argument shows that $S_{\lambda}(s)$ is the Laplace transform of a function $S_{\lambda}(t)$ and

$$
S_{\lambda}(t)=\frac{1}{2 \pi i} \int_{-i \infty}^{i \infty} e^{s t} \hat{S}_{\lambda}(s) d s .
$$

By the theorem of M. Riesz, if $2 \leqq p<\infty$ and $1 / q+1 / p=1$,

$$
\begin{aligned}
\left\|S_{\lambda}(t)\right\|_{L^{p}} & \leqq c\left[\int_{-i \infty}^{i \infty}\left|S_{\lambda}(s)\right|^{q}|d s|\right]^{1 / q} \\
& \leqq c\left[\int_{-i \infty}^{+\infty} \frac{|d s|}{(|\lambda|+|s|)^{q}}\right]^{1 / q} \leqq \frac{c}{|\lambda|^{1 / p}},
\end{aligned}
$$

where (2.13) has been used.

Let $C_{n}$ be the intersection of the contour $C$ of Lemma 3 with the disk $\{\lambda ;|\lambda| \leqq n\}$. Consider the function

$$
S_{n}(t)=\frac{1}{2 \pi i} \int_{C_{n}}(\lambda I-A)^{-1} S_{\lambda}(t) d \lambda .
$$

The operator $S_{\lambda}(t)$ can be shown to be analytic in $\lambda$ for $\lambda \in \bar{\Lambda}$ and any fixed $t>0$, but since this fact is not needed we omit the proof.

We claim that $\left\{S_{n}(t)\right\}$ is a Cauchy sequence in $L^{p}(0, \infty ; B(X))$ for any $2 \leqq p<\infty$. In fact, if $n>m$ and $C^{\prime}=C_{n} \mid C_{m}$,

$$
\begin{aligned}
\left\|S_{n}(t)-S_{m}(t)\right\|_{B(X)} & \leqq \frac{1}{2 \pi} \int_{C^{\prime}}\left\|(\lambda I-A)^{-1}\right\|_{B(X)}\left|S_{\lambda}(t)\right||d \lambda| \\
& \leqq c \int_{C^{\prime}}\left|S_{\lambda}(t)\right| \frac{|d \lambda|}{|\lambda|}
\end{aligned}
$$

Hence, by (2.15),

$$
\left\|S_{n}(t)-S_{m}(t)\right\|_{L^{p}} \leqq c \int_{C^{\prime}} \frac{|d \lambda|}{|\lambda|^{1+1 / p}} \rightarrow 0 \quad \text { if } n, m \rightarrow \infty .
$$

The limit in $L^{p}(0, \infty ; B(X))$ of the $S_{n}(t)$ is denoted by

$$
S(t)=\frac{1}{2 \pi i} \int_{C}(\lambda I-A)^{-1} S_{\lambda}(t) d \lambda .
$$

Since $S(t)$ is in $L^{p}(0, \infty ; B(X))$, it has a Laplace transform $S^{\wedge}(s)$, defined for $\operatorname{Re} s>0$. Using Fubini's theorem we get from (2.16),

$$
S^{\wedge}(s)=\frac{1}{2 \pi i} \int_{C}(\lambda I-A)^{-1} S_{\lambda}(s) d \lambda=[s I+g(s) A]^{-1},
$$

where the last equality follows from $[2$, p. 601]. 
We now prove that the function $S(t)$ defined by $(2.16)$ is a fundamental solution. From (2.17) we have:

$$
\left(I+h^{\wedge}(s) A\right) S^{\wedge}(s)=\frac{1}{s} I .
$$

Applying $A^{-1}$ to both sides of this equality, we get

$$
A^{-1} S^{\wedge}(s)+h^{\wedge}(s) S^{\wedge}(s)=A^{-1} / s .
$$

We shall now verify the relation

$$
A^{-1} S(t)+\int_{0}^{t} h(t-\tau) S(\tau)=A^{-1}
$$

Since $A^{-1}$ is a bounded operator and $S \in L^{p}(0, \infty ; B(X))$, the convolution theorem shows that the Laplace transforms of both sides of (2.20), for $\operatorname{Re} s>0$, coincide with the corresponding sides in (2.19). By the Corollary to Lemma 1 it follows that (2.20) is valid.

From (2.20) we see that the convolution $h * S$ is in the domain of $A$. Applying $A$ to both sides of (2.20), (1.1) follows.

To prove uniqueness, suppose $S_{0}(t)$ is another fundamental solution, and suppose that $e^{\alpha t} S_{0}(t) \in L^{r}(0, \infty ; B(X))$ for some $r \geqq 1, \alpha \geqq 0$. Writing (1.1) for $S_{0}$, applying $A^{-1}$ and then taking the Laplace transform, we get (2.19) for $S_{0}(s)$ when $\operatorname{Re} s>\alpha$. Applying $A$ to this equation we get (2.18) for $S_{0}^{\wedge}$. Hence $S^{\wedge}(s)$ $=S_{0}^{\wedge}(s)$ if $\operatorname{Re} s>\alpha$. By the Corollary to Lemma 1 it follows that $S(t)=S_{0}(t)$ almost everywhere.

REMARK. If $B(X)$ is a Hilbert space, the proof of Theorem 1 can be simplified. In fact, we define $S^{\wedge}(s)$ by (2.10), and using (2.5) and (iii $\left.{ }_{A}\right)$ we obtain

$$
\left\|S^{\wedge}(s)\right\| \leqq c /(1+|s|) .
$$

Since $B(X)$ is a Hilbert space, and since $S^{\wedge}(s)$ is analytic for $\operatorname{Re} s>0$ and (by (2.21)) in $L^{q}(1<q \leqq \infty)$ on vertical lines, we conclude that $S^{\wedge}(s)$ is the Laplace transform on operator function $S(t) \in L^{p}(0, \infty ; B(X))$ for any $2 \leqq p<\infty$, and

$$
\left[\int_{0}^{\infty}\|S(t)\|^{p} d t\right]^{1 / p} \leqq c\left[\int_{-i \infty}^{i \infty}\left\|S^{\wedge}(s)\right\|^{q}|d s|\right]^{1 / q}\left(\frac{1}{p}+\frac{1}{q}=1\right)
$$

One can now verify, as before, that $S(t)$ is a fundamental solution. When $B(X)$ is a Banach space, the theory of Fourier transform is not available to be applied to the operator $S^{\wedge}(s)$. Consequently, it is not possible to assert directly that $S^{\wedge}(s)$ is a Laplace transform. Thus we were forced to study the scalar functions $S_{\lambda}^{\wedge}(s)$ first and, for this purpose, to prove Lemmas 3 and 4.

3. $S$ in $L^{1}(0, \infty ; B(X))$. In this section we shall strengthen the conditions on $h$ and then prove that $S(t)$ belongs to $L^{p}(0, \infty ; B(X))$ for any $1 \leqq p<\infty$. Note that, by Hölder's inequality and Theorem 1 , if one proves that $S(t) \in L^{1}(0, \infty ; B(X))$ 
then also $S(t) \in L^{p}(0, \infty ; B(X))$ for $1<p<2$, and thus for all $1 \leqq p<\infty$. We shall also derive necessary conditions for the existence of a fundamental solution which is in $L^{1}(0, \infty ; B(X))$. For $h$ monotone decreasing and $\geqq 0$, the necessary conditions are precisely the conditions (2.1).

We need the following condition on $h$ :

(iii $\left.{ }_{\mathrm{h}}\right) \operatorname{th}(t) \in L^{1}(0, \infty)$.

TheOREM 2. Let $h \in \mathfrak{H}, A \in \mathfrak{A}$ and let $\left(\mathrm{iii}_{\mathrm{h}}\right)$ and (2.1) hold. Then there exists a fundamental solution $S(t)$ of $(1.1)$ in $L^{p}(0, \infty ; B(X))$ for all $1 \leqq p<\infty$.

Proof. All we have to prove is that the fundamental solution $S(t)$ which was constructed in $\S 2$ belongs to $L^{1}(0, \infty ; B(X))$.

We first extend Lemma 5:

Lemma 6. Let $h \in \mathfrak{S}, A \in \mathfrak{A}$ and let $\left(\mathrm{iii}_{\mathrm{h}}\right)$ and (2.1) hold. Then, in addition to the assertions of Lemma 5, we have:

$$
\left|\hat{S}_{\lambda}^{\prime}(s)\right| \leqq c|\lambda| /(|\lambda|+|s|)^{2} \quad \text { for } \operatorname{Re} s \geqq 0, \lambda \in \bar{\Lambda} .
$$

Proof. From (2.12) we have:

$$
S_{\lambda}^{\prime}(s)=-\left[1+\lambda g^{\prime}(s)\right]\left[\hat{S}_{\lambda}(s)\right]^{2} .
$$

A simple calculation shows that $g^{\prime}(s)$ is the Laplace transform of - $t h(t)$. Therefore, by (iii $\left.\mathrm{h}_{\mathrm{h}}\right), g^{\prime}(s)$ is a bounded function for $\operatorname{Re} s>0$. (3.1) now follows from (3.2) and (2.13).

LEMMA 7. Let the assumptions of Lemma 6 hold. Then

$$
\begin{array}{rlrl}
\left\|t S_{\lambda}(t)\right\|_{L^{p}} \leqq c /|\lambda|^{1 / p}, & & \text { if } 2 \leqq p<\infty \\
\left\|S_{\lambda}(t)\right\|_{L^{p}} \leqq c /|\lambda|^{1 / 2}, & & \text { if } 1 \leqq p \leqq 2 \\
& \leqq c /|\lambda|^{1 / p}, & & \text { if } 2<p<\infty .
\end{array}
$$

Proof. (3.4) with $2 \leqq p<\infty$ was proved in $\S 2$ (see (2.15)). Since $\hat{S}_{\lambda}^{\prime}(s)$ is the Laplace transform of $-t S_{\lambda}(t)$, we can proceed analogously to the paragraph following the proof of Lemma 5 and, using (3.1), obtain (3.3). Next,

$$
\begin{aligned}
\left\|S_{\lambda}(t)\right\|_{L^{1}} & \leqq\left[\int_{0}^{1}\left|S_{\lambda}(t)\right|^{2} d t\right]^{1 / 2}+\left[\int_{1}^{\infty} \frac{d t}{t^{2}}\right]^{1 / 2}\left[\int_{1}^{\infty}\left|t S_{\lambda}(t)\right|^{2} d t\right]^{1 / 2} \\
& \leqq\left\|S_{\lambda}(t)\right\|_{L^{2}}+\left\|t S_{\lambda}(t)\right\|_{L^{2}} \leqq \frac{c}{|\lambda|^{1 / 2}}
\end{aligned}
$$

where we have used (2.15) and (3.3). Having proved (3.4) for $p=1$, the result for $1<p<2$ follows from the results for $p=1$ and $p \geqq 2$, by using Hölder's inequality.

Using (3.4) we can now proceed as in $\$ 2$ to show that $S(t)$ is the limit in $L^{p}(0, \infty ; B(X))$ of the $S_{n}(t)$, for any $1 \leqq p<2$. Thus $S(t) \in L^{p}(0, \infty ; B(X))$ and the proof is complete. 
We next prove a theorem which shows that the condition (2.2) and a part of the condition (2.3) are necessary for $S(t)$ to belong to $L^{1}(0, \infty ; B(X))$.

THEOREM 3. Let $h \in \mathfrak{L}, A \in \mathfrak{A}$ and assume that there exists a fundamental solution $S(t)$ of $(1.1)$ in $L^{1}(0, \infty ; B(X))$. Then (2.2) holds and $g(0) \neq 0$.

Proof. To prove that $g(0) \neq 0$ we use (2.19) (which follows from (2.18)). Multiplying both sides by $s$ and taking $s \rightarrow 0$, we get (since $S^{\wedge}(s)$ is continuous in $s$ )

$$
g(0) S^{\wedge}(0)=A^{-1} \text {; }
$$

hence $g(0) \neq 0$. To prove (2.2), note that $S^{\wedge}(s)$ must satisfy $(2.18)$ and must be a uniformly bounded operator for $\operatorname{Re} s \geqq 0$. Writing (2.18) in the form

$$
(\lambda I-A) g(s) S^{\wedge}(s)=-I \quad\left(\lambda=-1 / h^{\wedge}(s)\right)
$$

and recalling that $g(s)$ is a bounded function for $\operatorname{Re} s \geqq 0$, we conclude that for each point $\lambda \in \Delta \cap \rho(A)$

$$
\left\|(\lambda I-A)^{-1}\right\| \leqq c \quad(c \text { is independent of } \lambda) .
$$

Hence there exists a disk about $\lambda$, with radius $\delta$ independent of $\lambda$, which is contained in $\rho(A)$. Thus the set $\Delta \cap \rho(A)$ is closed and open in $\Delta$. If we show that it also nonempty then, since $\Delta$ is a closed domain, the set $\Delta \cap \rho(A)$ must coincide with $\Delta$, i.e., $\Delta \subset \rho(A)$. Now, in the first steps of the proof of Lemma 3 it was shown that if $|s|$ is sufficiently large and $\operatorname{Re} s \geqq 0$, then $\lambda=-1 / h^{\wedge}(s)$ belongs to $\rho(A)$; thus, in fact, $\Delta \cap \rho(A)$ is nonempty.

Consider the case where $h(t)$ is monotone decreasing and nonnegative. Then, by (1.2),

$$
|g(s)| \geqq h(0)-\int_{0}^{\infty}\left|e^{-s t} h(t)\right| d t \geqq h(0)+\int_{0}^{\infty} h(t) d t=h(\infty)
$$

if $\operatorname{Re} s \geqq 0$, and

$$
g(0)=h(0)+\int_{0}^{\infty} h(t) d t=h(\infty) .
$$

Hence (2.3) holds if and only if $g(0) \neq 0$, i.e., if and only if $h(\infty) \neq 0$. Combining Theorems 2, 3 we thus obtain:

THEOREM 4. Let $A \in \mathfrak{A}$ and let $h$ be a nonnegative monotone decreasing function in $\mathfrak{S}$, satisfying (iii $\left.\mathrm{h}_{\mathrm{h}}\right)$. Then there exists a fundamental solution $S(t)$ of $(1.1)$ in $L^{1}(0, \infty ; B(X))$ if and only if (2.1) holds, or if and only if (2.2) holds and $h(\infty)>0$.

4. Further bounds on $S$. In this section we replace the condition ( $\mathrm{iii}_{\mathrm{h}}$ ) of $\S 3$ by the stronger condition:

$\left(\mathrm{iii}_{\mathrm{h}}{ }^{\prime}\right) \operatorname{th}(t) \in L^{1}(0, \infty)$ and, for some $p$ with $1<p \leqq 2, h(t) \in L^{p}(0, \infty)$ and

$$
\int_{-\infty}^{\infty}|h(\tau+t)-h(\tau-t)|^{p} d \tau=O(t) \quad \text { as } t \rightarrow 0
$$


We shall then prove that $S(t)$ is continuous and converges to 0 as $t \rightarrow \infty$. We shall also get an estimate on $A^{\rho} S$ for $0<\rho \leqq 1$, which will be needed in $\S 6$.

LEMMA 8. Let $h \in \mathfrak{S}$ and let (iii $\left.{ }^{\prime}{ }^{\prime}\right)$ hold. Then $h(t)$ has a Laplace transform $h^{\wedge}(s) \in H^{q}(0$, C) for all $1<q \leqq \infty$.

Proof. Since $h(t) \in L^{1}, h^{\wedge}(s) \in H^{\infty}(0, \mathrm{C})$. By [19, Theorem 84], we have that $h^{\wedge}(s) \in H^{q}(0, \mathrm{C})$ for every $1<q \leqq p /(p-1)$. Now use Hölder's inequality to get the assertion for any $1<q \leqq \infty$.

ThEOREM 5. Let $h \in \mathfrak{S}, A \in \mathfrak{A}$ and let (2.1) and (iii $\left.{ }^{\prime}\right)$ hold. Then $S(t)$ is continuous for $t \geqq 0$ and its norm approaches zero as $t \rightarrow \infty$.

Proof. Let $E(t)$ be the fundamental solution of $d v / d t+h(0) A v=0$ (see $\S 1.2$ ). Using (2.13) and the first equation in (2.17), we get

$$
\left\|S^{\wedge}(s)\right\| \leqq c \int_{C}\left|S_{\lambda}(s)\right| \frac{|d \lambda|}{|\lambda|} \leqq \frac{c}{1+|s|} \quad \text { if } \operatorname{Re} s \geqq 0
$$

By [8], $E^{\wedge}(s)$ exists and is given by

$$
E^{\wedge}(s)=[s I+h(0) A]^{-1} \text {. }
$$

Using the second relation in (2.17) we then obtain

$$
\begin{aligned}
S^{\wedge}(s) & =E^{\wedge}(s)-[g(s)-h(0)] A E^{\wedge}(s) S^{\wedge}(s) \\
& =E^{\curlywedge}(s)-h^{\wedge}(s) A E^{\curlywedge}(s) S^{\wedge}(s),
\end{aligned}
$$

where the facts that the operators $E^{\wedge}, S^{\wedge}, A E^{\wedge}$ and $A S^{\wedge}$ (by (2.17)) are bounded and commute with each other have been used. We can thus write

$$
S^{\wedge}(s)=E^{\wedge}(s)-T^{\wedge}(s)
$$

where

$$
\left\|T^{\wedge}(s)\right\| \leqq\left|h^{\wedge}(s)\right|\left\|A E^{\wedge}(s)\right\|\left\|S^{\wedge}(s)\right\| \leqq(c /(1+|s|))\left|h^{\wedge}(s)\right| ;
$$

here we used (4.2) and the fact that $\left\|A E^{\wedge}(s)\right\| \leqq c$.

Since, by Lemma $8,\left|h^{\wedge}(s)\right| \in L^{2}$ on vertical lines $\operatorname{Re} s=\alpha \geqq 0,\left\|T^{\wedge}(s)\right\|$ belongs to $L^{1}$ on these vertical lines. Hence $T^{\wedge}(s) \in H^{1}(0 ; B(X))$. By Lemma 2 it is therefore the Laplace transform of a continuous operator-valued function $T(t)$, and $\|T(t)\| \rightarrow 0$ as $t \rightarrow \infty$.

From (4.4) and the Corollary to Lemma 1 we get:

$$
S(t)=E(t)-T(t)
$$

and since (see [8]) $E(t)$ is continuous in $t$ and $\|E(t)\| \rightarrow 0$ as $t \rightarrow \infty$, the proof is complete. 
TheOREM 6. Let $h \in \mathfrak{H}, A \in \mathfrak{A}$ and let (2.1) and $\left(\mathrm{iii}_{\mathrm{h}}{ }^{\prime}\right)$ hold. Then, for any $0 \leqq \rho \leqq 1$, $A^{\rho} S(t)$ is a bounded operator for $t>0$, and

$$
\left\|A^{\rho} S(t)\right\| \leqq c / t^{\circ} .
$$

Proof. By (2.17),

$$
\begin{aligned}
\left\|A S^{\wedge}(s)\right\| & =\left\|A[s I+g(s) A]^{-1}\right\| \\
& =\left\|\left[I-\left(I+h^{\wedge}(s) A\right)^{-1}\right] / g(s)\right\|=\left\|\left[I-s S^{\wedge}(s)\right] / g(s)\right\| .
\end{aligned}
$$

Using (2.14), (4.2), we then get

$$
\left\|A S^{\wedge}(s)\right\| \leqq c .
$$

Hence, by (1.5.5) with $A(t)=A, v=S^{\wedge}(s)$,

$$
\left\|A^{\rho} S^{\wedge}(s)\right\| \leqq c /(1+|s|)^{1-\rho} .
$$

Next, from the definition of $A^{-\rho}$ and from (4.3) it follows that $A^{-\rho}$ commutes with $E^{\wedge}$. Hence $A^{1+\rho} E^{\wedge} A^{-\rho}=A E^{\wedge}$. It follows that $A^{1+\rho} E^{\wedge} \supset A E^{\wedge} A^{\rho}$. Since $A E^{\wedge} A^{\rho} S^{\wedge}$ is a bounded operator, we then obtain the equality

$$
A^{1+\rho} E^{\wedge} S^{\wedge}=A E^{\wedge} A^{\rho} S^{\wedge} \text {. }
$$

Hence (4.4) gives

$$
A^{\rho} S^{\wedge}(s)-A^{\rho} E^{\wedge}(s)=-h^{\wedge}(s) A E^{\wedge}(s) A^{\rho} S^{\wedge}(s) \equiv G(s) .
$$

Noting, by (4.3) and ( $\left.\mathrm{iii}_{\mathrm{A}}\right)$, that $\left\|A E^{\wedge}(s)\right\| \leqq c$ and using (4.8), we then obtain

$$
\|G(s)\| \leqq\left(c /(1+|s|)^{1-\rho}\right)\left|h^{\wedge}(s)\right| .
$$

By Lemma 8 , the right-hand side belongs to $L^{1}$ on any vertical line $\operatorname{Re} s=\alpha$ with $\alpha \geqq 0$. Observing next, by (4.3), that $A E^{\wedge}(s)$ is analytic in $s$ for $\operatorname{Re} s \geqq 0$, and, by (2.17), that $A S^{\wedge}(s)$ (and, therefore, $A^{\rho} S^{\wedge}(s)$ ) is analytic in $s$ for $\operatorname{Re} s \geqq 0$, we see that $G(s)$ is analytic in $s$ for $\operatorname{Re} s \geqq 0$. We can therefore apply Lemma 2 and conclude that there exists a bounded operator $H(t)$ such that $H^{\wedge}(s)=G(s)$. Hence also $\left(A^{-\rho} H\right)^{\wedge}(s)=A^{-\rho} G(s)$.

Writing (4.9) in the form

$$
S^{\wedge}(s)-E^{\wedge}(s)=A^{-\rho} G(s)
$$

and applying the Corollary to Lemma 1 we get

$$
S(t)-E(t)=A^{-\rho} H(t) .
$$

Hence,

$$
\left\|A^{\rho} S(t)-A^{\rho} E(t)\right\|=\|H(t)\| \leqq c .
$$

Since, finally, $\left\|A^{\rho} E(t)\right\| \leqq c / t^{\rho}$, (4.7) follows. 
5. Further bounds on $S$ (continued).

DEFINITION. We say that $h$ belongs to the class $\mathfrak{S}_{n}$ if $h$ satisfies the conditions $\left(\mathrm{i}_{\mathrm{h}}\right)$, (ii $\left.\mathrm{i}_{\mathrm{h}}\right)$ of $\S 1$ (i.e., if $h \in \mathfrak{S}$ ) and the additional conditions:

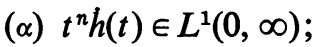

( $\beta$ ) for some $p$, with $1<p \leqq 2, h(t) \in L^{p}(0, \infty)$ and (4.1) holds. If instead of $(\alpha)$ we have:

$\left(\alpha^{\prime}\right) e^{v t} h(t) \in L^{1}(0, \infty)$ for some $\nu>0$, then we say that $h$ belongs to $\mathfrak{S}_{\infty}$.

The results of $\S 4$ were obtained under the assumption that $h \in \mathfrak{H}_{1}$. In this section we assume that $h \in \mathfrak{S}_{n}$ or $h \in \mathfrak{S}_{\infty}$ and obtain additional bounds on $S$.

TheOREM 7. Let $h \in \mathfrak{S}_{n}, A \in \mathfrak{A}$ and let (2.1) hold. Then

$$
\left(1+t^{n-1}\right) S(t) \in L^{1}(0, \infty ; B(X)) .
$$

Proof. We first prove by induction on $k(0 \leqq k \leqq n)$ that

$$
\left|\hat{S}_{\lambda}^{(k)}(s)\right| \leqq c \sum_{j=0}^{k}|\lambda|^{j} /(|\lambda|+|s|)^{j+1} \quad(c=c(k)) .
$$

For $k=0$, this is just (2.13). Assuming that (5.1) holds for all $k \leqq n-1$ we shall prove it for $k=n$.

Differentiating (3.2) $n-1$ times we get,

$$
\begin{aligned}
\hat{S}_{\lambda}^{(n)}= & -\left(1+\lambda g^{\prime}\right)\left(\hat{S}_{\lambda}^{2}\right)^{(n-1)}-\lambda \sum_{j=0}^{n-2}\left(\begin{array}{c}
n-1 \\
j
\end{array}\right) g^{(n-j)}\left(\hat{S}_{\lambda}^{2}\right)^{(j)} \\
= & -\left(1+\lambda g^{\prime}\right) \sum_{m=0}^{n-1}\left(\begin{array}{c}
n-1 \\
m
\end{array}\right) \hat{S}_{\lambda}^{(n-1-m)} \hat{S}_{\lambda}^{(m)} \\
& -\lambda \sum_{j=0}^{n-2}\left(\begin{array}{c}
n-1 \\
j
\end{array}\right) g^{(n-j)} \sum_{m=0}^{j}\left(\begin{array}{c}
j \\
m
\end{array}\right) \hat{S}_{\lambda}^{(j-m)} \hat{S}_{\lambda}^{(m)} .
\end{aligned}
$$

From $g(s)=h(0)+h^{\wedge}(s)$ we derive, by a simple calculation,

$$
g^{(j)}=(-1)^{j}\left(t^{j} h\right)^{\wedge} \quad(1 \leqq j \leqq n)
$$

and by our assumptions on $h$ we conclude that $\left|g^{(j)}(s)\right| \leqq c$ for all $s$ with $\operatorname{Re} s \geqq 0$. Using this fact and the inductive assumption (5.1) for $0 \leqq k \leqq n-1$, we obtain from (5.2) the inequality (5.1) with $k=n$.

We can now proceed similarly to the proof of Lemma 7 , noticing that $\hat{S}_{\lambda}^{(m)}(s)$ is (for $0 \leqq m \leqq n)$ the Laplace transform of $(-1)^{m} t^{m} S_{\lambda}(t)$. We obtain

$$
\left\|t^{m} S_{\lambda}(t)\right\|_{L^{2}} \leqq\left\|\hat{S}_{\lambda}^{(m)}(s)\right\|_{L^{2}} \leqq c /|\lambda|^{1 / 2} .
$$

Using this result for $m=n, m=n-1$ and arguing as in the proof of Lemma 7, we get

$$
\left\|t^{n-1} S_{\lambda}(t)\right\|_{L^{1}} \leqq c /|\lambda|^{1 / 2}
$$


Employing this inequality, we obtain from (2.16):

$$
\left\|t^{n-1} S(t)\right\|_{L^{1}} \leqq c \int_{C}\left\|t^{n-1} S_{\lambda}(t)\right\|_{L^{1}} \frac{|d \lambda|}{|\lambda|} \leqq c \int_{C} \frac{|d \lambda|}{|\lambda|^{3 / 2}}<\infty
$$

and the proof is complete.

In the previous proof we have only derived an analog of Lemma 7 with $p=2$. The analogous results for $p \geqq 2$ can also be obtained in the same way. This leads to the following:

Corollary. Let $h \in \mathfrak{S}_{n}, A \in \mathfrak{A}$ and let (2.1) hold. Then

$$
\left(1+t^{n}\right) S(t) \in L^{p}(0, \infty ; B(X))
$$

for every $p$ with $2 \leqq p<\infty$.

THEOREM 8. Let $h \in \mathfrak{S}_{\infty}, A \in \mathfrak{A}$ and let (2.1) hold. Then $e^{\alpha t} S(t) \in L^{\infty}(0, \infty ; B(X))$ for some $\alpha>0$.

Proof. We already know that $S(t) \in L^{\infty}(0, \infty ; B(X))$, that $S^{\wedge}(s)$ is analytic for $\operatorname{Re} s \geqq 0$, and that it satisfies (4.2). If we can prove that $S^{\wedge}(s)$ is analytic for $\operatorname{Re} s \geqq-\alpha$ and

$$
\left\|S^{\wedge}(s)\right\| \leqq c /(1+|s|) \quad \text { for } \operatorname{Re} s \geqq-\alpha
$$

then, by Lemma $2, S^{\wedge}(s-\alpha)$ (for $\operatorname{Re} s \geqq 0$ ) will be the Laplace transform of

$$
f(t) \equiv \int_{\gamma-i \infty}^{\gamma+i \infty} e^{s t} S^{\wedge}(s-\alpha) d s=e^{\alpha t} \int_{\delta-i \infty}^{\delta+i \infty} e^{s t} S^{\wedge}(s) d s
$$

for any $\gamma \geqq 0, \delta=\gamma-\alpha$, and $f(t)$ will be a bounded function. Since the integral on the right-hand side is equal to $S(t)$, it will follow that $e^{\alpha t}\|S(t)\| \leqq c$.

It remains to prove the analyticity of $S^{\wedge}(s)$ in $\operatorname{Re} s \geqq-\alpha$ and the inequality (5.3).

Writing

$$
S^{\wedge}(s)=\left[\left(1 / h^{\wedge}(s)\right) I+A\right]^{-1} / g(s)
$$

for $\operatorname{Re} s \geqq 0$, we first have to prove that

$$
-1 / h^{\wedge}(s) \in \rho(A), \quad \text { if } \operatorname{Re} s \geqq-\alpha .
$$

We have

$$
s h^{\wedge}(s)=h(0)+h^{\wedge}(s)=h(0)+o(1) \quad \text { in the half-plane } \operatorname{Re} s \geqq-\nu .
$$

Hence, as in the first steps of the proof of Lemma 3,

$$
-1 / h^{\wedge}(s) \in \rho(A), \quad \text { if } \operatorname{Re} s \geqq-\nu \text { and }|s| \geqq s_{\nu}
$$


On the other hand, if $|s| \leqq s_{v}$ and $\operatorname{Re} s=0$ then $-1 / h^{\wedge}(s)$ lies in $\rho(A)$ and, consequently, by continuity,

$$
-1 / h^{\wedge}(s) \in \rho(A), \quad \text { if } \operatorname{Re} s \geqq-\alpha, \quad|s| \leqq s_{\nu}
$$

provided $\alpha$ is sufficiently small. Thus (5.5) is valid.

Next we observe that $g(s)$ is analytic for $\operatorname{Re} s \geqq-\nu$. We claim that

$$
g(s) \neq 0, \quad \text { if } \operatorname{Re} s \geqq-\alpha
$$

for some sufficiently small $\alpha>0$ (which, for simplicity, we take to be the same as in (5.5)). Indeed, if this were not the case, there would exist a sequence $\left\{s_{n}\right\}$ with $\operatorname{Re} s_{n} \rightarrow 0$ such that $g\left(s_{n}\right)=0$. If $\left|s_{n}\right| \rightarrow \infty$ then $g\left(s_{n}\right) \rightarrow h(0) \neq 0$, which is a contradiction. If, on the other hand, $s_{n} \rightarrow s^{*}$ then, by continuity, $g\left(s^{*}\right)=0$, thus contradicting (2.3).

We conclude from (5.4) that $S^{\wedge}(s)$ is analytic in $\operatorname{Re} s \geqq-\alpha$. Furthermore, using (iii $\left.{ }_{A}\right)$ we obtain (5.3).

\section{Representation of solutions.}

Definition. A function $k(t)$ is said to belong to the class $\AA_{p, \mu}$ where $1 \leqq p \leqq \infty$, $0<\mu \leqq 1$, if $k(t) \in C^{1}([0, \infty) ; X), \dot{k}(t) \in L^{p}(0, \infty ; X), k(0) \in D\left(A^{\mu}\right), \dot{k}(t) \in D\left(A^{\mu}\right)$ and $A^{\mu} \dot{k}(t) \in L^{1}(0, T ; X)$ for any $T>0$.

DEFINITION. A function $u(t)$ is called an $L^{a}$-solution of

$$
u(t)=k(t)-\int_{0}^{t} h(t-\tau) A u(\tau) d \tau
$$

if $u(t) \in L^{q}(0, \infty ; X), u(t) \in D(A)$ for almost all $t>0, A u(t) \in L^{1}(0, T ; X)$ for all $T>0$, and (6.1) holds for almost all $t>0$. If the assumption $u \in L^{q}(0, \infty ; X)$ is omitted, then we simply call $u$ a solution of (6.1).

TheOREM 9. Let $h \in \mathfrak{S}_{1}, A \in \mathfrak{A}, k \in \mathfrak{R}_{p, \mu}$, and let (2.1) hold. Then the function

$$
u(t)=S(t) k(0)+\int_{0}^{t} S(t-\tau) \dot{k}(\tau) d \tau
$$

is an $L^{q}$-solution of (6.1) for any $q$ with $p \leqq q \leqq \infty$. Furthermore, $u(t) \in C^{0}([0, \infty) ; X)$ and, if $p<\infty, u(t) \rightarrow 0$ as $t \rightarrow \infty$. If $v$ is any $L^{q}$-solution for some $1 \leqq q \leqq \infty$, then $v(t)=u(t)$ for almost all $t>0$.

Proof. By Theorems $5,2, S(t) k(0)$ is continuous and bounded for $0 \leqq t<\infty$, approaches zero as $t \rightarrow \infty$, and belongs to $L^{r}(0, \infty ; X)$ for any $1 \leqq r \leqq \infty$.

Next, by Theorems 5, 2 and Young's inequality [19] we find that the second integral on the right-hand side of (6.2) is continuous for $0 \leqq t<\infty$, belongs to $L^{q}(0, \infty ; X)$ for any $p \leqq q \leqq \infty$ and, if $p<\infty$, approaches zero as $t \rightarrow \infty$.

We proceed to prove that $u$ is an $L^{q}$-solution. Taking the Laplace transform of both sides of (6.2) we get

$$
u^{\wedge}(s)=S^{\wedge}(s)\left[k(0)+\dot{k}^{\wedge}(s)\right], \quad \text { if } \operatorname{Re} s>0 .
$$


Thus, by (2.18),

$$
\left[I+h^{\wedge}(s) A\right] u^{\wedge}(s)=\frac{1}{s}\left[k(0)+\dot{k}^{\wedge}(s)\right]=k^{\wedge}(s),
$$

and

$$
\left[A^{-1}+h^{\wedge}(s) I\right] u^{\wedge}(s)=A^{-1} k^{\wedge}(s) .
$$

Since $A^{-1}$ is a bounded operator, $A^{-1} u^{\wedge}(s)$ is the Laplace transform of $A^{-1} u(t)$. The same remark applies to $k$. Hence, by the convolution theorem and the Corollary to Lemma 1, we conclude from (6.3) that

$$
A^{-1} u(t)+\int_{0}^{t} h(t-\tau) u(\tau) d \tau=A^{-1} k(t)
$$

Consequently the integral $\int_{0}^{t} h(t-\tau) u(\tau) d \tau$ belongs to $D(A)$ and

$$
u(t)+A \int_{0}^{t} h(t-\tau) u(\tau) d \tau=k(t)
$$

To prove that $A$ commutes with the integral in (6.5), we begin with the relation

$$
A^{\mu} \int_{0}^{t} S(t-\tau) \dot{k}(\tau) d \tau=\int_{0}^{t} A^{\mu} S(t-\tau) \dot{k}(\tau) d \tau
$$

which follows by Theorem 6 and the fact that $A^{\mu}$ is closed. Next, from (2.18) it is clear that $A^{-\mu} S^{\wedge}=S^{\wedge} A^{-\mu}$. Applying the Corollary to Lemma 1 , we obtain $A^{-\mu} S=S A^{-\mu}$. Hence

$$
A^{\mu} S(t) \supset S(t) A^{\mu} .
$$

Using the fact that $\dot{k}(\tau) \in D\left(A^{\mu}\right)$ and (6.7), we get from (6.6)

$$
A^{\mu} \int_{0}^{t} S(t-\tau) \dot{k}(\tau) d \tau=\int_{0}^{t} S(t-\tau) A^{\mu} \dot{k}(\tau) d \tau
$$

We also have

$$
A^{\mu} S(t) k(0)=S(t) A^{\mu} k(0)
$$

Again using Theorem 6 and the fact that $A^{1-\mu}$ is closed, we see that the righthand side of (6.8) belongs to the domain of $A^{1-\mu}$, and $A^{1-\mu}$ can be applied by introducing it inside the integral. Since the right-hand side of (6.9) also belongs to $D\left(A^{1-\mu}\right)$, we conclude that the right-hand side of (6.2) (i.e., $\left.u(t)\right)$ is in $D(A)$ and

$$
A u(t)=A^{1-\mu} S(t) \cdot A^{\mu} k(0)+\int_{0}^{t} A^{1-\mu} S(t-\tau) \cdot A^{\mu} \dot{k}(\tau) d \tau .
$$

Hence,

$$
\|A u(t)\| \leqq \frac{c}{t^{1-\mu}}\left\|A^{\mu} k(0)\right\|+c \int_{0}^{t}\left\|A^{\mu} \dot{k}(\tau)\right\| \frac{d \tau}{(t-\tau)^{1-\mu}}
$$


Therefore,

$$
\begin{aligned}
\int_{0}^{T}\|A u(t)\| d t & \leqq c T^{\mu}\left\|A^{\mu} k(0)\right\|+c \int_{0}^{T}\left\|A^{\mu} \dot{k}(\tau)\right\| \int_{\tau}^{T} \frac{d t}{(t-\tau)^{1-\mu}} d \tau \\
& \leqq c T^{\mu}\left\|A^{\mu} k(0)\right\|+c(T)\left\|A^{\mu} \dot{k}(\tau)\right\|_{L^{1}(0, T ; X) .}
\end{aligned}
$$

Thus $A u(t) \in L^{1}(0, T ; X)$ for every $T>0$. Since $A$ is closed, we can then introduce the $A$ in (6.5) inside the integral and conclude that $u$ is indeed an $L^{q}$-solution.

To prove the last assertion of the theorem, suppose $v$ is an $L^{q}$-solution of (6.1) with $k \equiv 0$. Then it has a Laplace transform satisfying

$$
\left[I+h^{\wedge}(s) A\right] v^{\wedge}(s)=0 \text { for } \operatorname{Re} s,>0 .
$$

By (2.2) it follows that $v^{\wedge}(s)=0$ if $\operatorname{Re} s>0$ and, by the Corollary to Lemma 1, we have $v(t)=0$ for almost all $t>0$.

We remark that a simple consequence is the following well-known result:

Corollary. Let $h(t) \equiv 1, A \in \mathfrak{A}, k \in \mathfrak{\Re}_{p, \mu}$. Then the assertion of Theorem 9 is valid.

Indeed, we only have to verify (2.1), and this is clear since $\Delta=\{s ; \operatorname{Re} s \leqq 0\}$ and $g(s) \equiv 1$.

Definition. A function $k(t)$ is said to belong to the class $\mathfrak{R}_{\mu}$ if

$$
k(t) \in C^{1}([0, \infty) ; X), k(0) \in D\left(A^{\mu}\right), \dot{k}(t) \in D\left(A^{\mu}\right) \quad \text { and } \quad A^{\mu} \dot{k}(t) \in L^{1}(0, T ; X)
$$

for every $T>0$.

Corollary 2. Let $h \in \mathfrak{H}_{1}, A \in \mathfrak{A}, k \in \mathfrak{I}_{\mu}$, and let (2.1) hold. Then the right-hand side of (6.2) is a solution of (6.1).

We shall call this solution the local $L^{\infty}$-solution of (6.1).

Proof. It suffices to prove the assertion for $t \in\left[0, t_{0}\right]$ with any $t_{0}>0$. Modify $k(t)$ for $t>t_{0}$ so that the modified function $k_{0}(t)$ is in $\mathbb{R}_{1, \mu}$. The corresponding $L^{\infty}$-solution $u_{0}(t)$ (given by (6.2) with $k$ replaced by $k_{0}$ ) satisfies (6.1) for all $t>0$ and it coincides with $u(t)$ for $t \in\left[0, t_{0}\right]$. Hence $u(t)$ satisfies (6.1) for $t \in\left[0, t_{0}\right]$.

We conclude this section by noting that the uniqueness of solutions can be proved (by the same method as before) within the class of functions $v(t)$ which satisfy: $\|v(t)\|=O\left(e^{\gamma t}\right)$ for some $\gamma>0$. In the next section we shall prove a local uniqueness theorem.

\section{A uniqueness theorem and its applications.}

THEOREM 10. Let $h \in \mathfrak{S}_{1}, A \in \mathfrak{A}$ and let (2.2) hold. Let

$$
u_{i}(t)=k_{i}(t)-\int_{0}^{t} h(t-\tau) A u_{i}(\tau) d \tau \quad(i=1,2)
$$

for almost all $t>0$, where

$$
e^{-\alpha t} k_{i}(t), \quad e^{-\alpha t} u_{i}(t) \in L^{1}(0, \infty ; X)
$$


for some $\alpha>0$, and $A u_{i}(t) \in L^{1}\left(0, t_{0} ; X\right)$ for any $t_{0}>0$. If

$$
k_{1}(t)=k_{2}(t) \quad \text { for } 0 \leqq t \leqq T \quad(T>0),
$$

then $u_{1}(t)=u_{2}(t)$ for almost all $t \in[0, T]$.

Proof. Set $k=k_{1}-k_{2}, u=u_{1}-u_{2}$. These functions have Laplace transforms for $\operatorname{Re} s>\alpha$, continuous for $\operatorname{Re} s=\alpha$. From (7.1) we obtain

$$
u^{\wedge}(s)=\left[I+h^{\wedge}(s) A\right]^{-1} k^{\wedge}(s) \text {. }
$$

From (iii $\left.{ }_{\mathrm{A}}\right),(2.2)$ we get $\left\|\left[I+h^{\wedge}(s) A\right]^{-1}\right\| \leqq c$ if $\operatorname{Re} s \geqq 0$, while, by our assumptions on the $k_{i},\left\|k^{\wedge}(s)\right\|$ is bounded if $\operatorname{Re} s \geqq \alpha$. Therefore

$$
u^{\wedge}(s) /(1+s)^{2}=\left(1 /(1+s)^{2}\right)\left[I+h^{\wedge}(s) A\right]^{-1} k^{\wedge}(s) \in H^{1}(\alpha ; X) .
$$

Furthermore we have

$$
k^{\wedge}(s)=\int_{T}^{\infty} e^{-s t} k(t) d t=e^{-s T} \int_{0}^{\infty} e^{-s t} k(t+T) d t
$$

so that

$$
\left\|k^{\wedge}(s)\right\|=O\left(\left|e^{-s T}\right|\right) \quad \text { as }|s| \rightarrow \infty \text { in the half plane } \operatorname{Re} s \geqq \alpha .
$$

Now, by Lemma 2,

$$
\left[\frac{u^{\wedge}(s)}{(1+s)^{2}}\right]^{\curlyvee}=\frac{1}{2 \pi i} \int_{\beta-i \infty}^{\beta+i \infty} \frac{e^{s t}}{(1+s)^{2}}\left[I+h^{\wedge}(s) A\right]^{-1} k^{\wedge}(s) d s
$$

where " $\checkmark$ " denotes the inverse Laplace transform and $\beta \geqq \alpha$. By Cauchy's theorem we can write the last integral in the form

$$
\lim _{N \rightarrow \infty} \int_{\beta-i N}^{\beta+i N}=\lim _{N \rightarrow \infty} \int_{C_{N}}
$$

where $C_{N}$ is a half circle in $\operatorname{Re} s \geqq \beta$ with the interval $[\beta-i N, \beta+i N]$ as diameter. Using (7.2) we see that if $t<T$ then the right-hand side of (7.3) is zero. Hence,

$$
\left[u^{\wedge}(s) /(1+s)^{2}\right]^{\curlyvee}=0 \text { for } t<T \text {. }
$$

But then

$$
\left[u^{\wedge}(s) /(1+s)^{2}\right]^{\curlyvee}=\int_{0}^{t}(t-\tau) e^{-(t-\tau)} u(\tau) d \tau=0 \quad \text { if } t<T .
$$

Differentiating this relation we get

$$
\int_{0}^{t}\left[e^{-(t-\tau)}-(t-\tau) e^{-(t-\tau)}\right] u(\tau) d \tau=0,
$$

so that

$$
\int_{0}^{t} e^{\tau} u(\tau) d \tau=0 \quad \text { if } t<T,
$$

i.e., $u(t)=0$ for almost all $t \in[0, T]$. 
Definition. We say that $h$ belongs to the class $\mathfrak{S}^{\prime}$ if $h \in \mathfrak{S}_{1}$ and if, in addition, $h \in C^{2}[0, \infty), \breve{h}(t)$ is uniformly Hölder continuous in closed subsets of $[0, \infty)$ and $\grave{h}(t)=O\left(e^{\gamma t}\right)$ for some $\gamma>0$.

Definition. We say that $k$ belongs to the class $\mathfrak{\Omega}_{\mu}^{\prime}$ if $k \in \mathfrak{\Re}_{\mu}$ and if, in addition, $\dot{k}(t)$ is uniformly Hölder continuous in closed subsets of $[0, \infty)$.

TheOREM 11. Let $h \in \mathfrak{S}^{\prime}, A \in \mathfrak{A}, k \in \mathfrak{\Re}_{\mu}^{\prime}$, and let (2.1) hold. Then the local $L^{\infty}$ solution of (6.1) coincides with the strong solution of (6.1) for all $t>0$.

Proof. The local $L^{\infty}$-solution was defined in $\S 6$; denote it by $v(t)$. The strong solution of (6.1) was constructed in Theorem 1.1; denote it by $u(t)$. Let $k_{0}(t)$ be a function which belongs to $\mathfrak{\Re}_{\mu}^{\prime}$, vanishes for $t>T+1$, and coincides with $k(t)$ for $0 \leqq t \leqq T$. Denote by $u_{0}(t)$ the corresponding strong solution, and by $v_{0}(t)$ the corresponding $L^{1}$-solution of Theorem 9. Then, by Theorems 1.1, 9,

$$
\begin{aligned}
& u(t)=u_{0}(t), \quad \text { for } 0 \leqq t \leqq T, \\
& v(t)=v_{0}(t), \quad \text { for } 0 \leqq t \leqq T .
\end{aligned}
$$

Next, by Corollary 2 to Theorem 1.5 ,

$$
u_{0}(t)=W(t) k(0)+\int_{0}^{t} W(t-\tau) \dot{k}_{0}(\tau) d \tau \quad(W(t)=W(t, 0)) .
$$

Using Theorem 1.6 we see that $\left\|u_{0}(t)\right\|=O\left(e^{\delta t}\right)$ for some $\delta>0$.

Since $v_{0}(t) \in L^{1}(0, \infty ; X)$, we can apply Theorem 10 and conclude that $v_{0}(t)=u_{0}(t)$ for $0 \leqq t \leqq T$. Hence, by (7.4), $v(t)=u(t)$ for $0 \leqq t \leqq T$ and, since $T$ is arbitrary, $v(t)=u(t)$ for all $t>0$.

Taking $k(t) \equiv k(0)$ and comparing the representations (1.1.9), (6.2) for the strong solution and the $L^{1}$-solution respectively, we conclude:

Corollary 1. Let $h \in \mathfrak{S}^{\prime}, A \in \mathfrak{A}$ and let (2.1) hold. Then

$$
S(t) \equiv W(t) \quad(0 \leqq t<\infty) .
$$

From this and Corollary 2 to Theorem 1.5 we get:

Corollary 2. Let $h \in \mathfrak{S}^{\prime}, A \in \mathfrak{A}$ and let (2.1) hold. Assume that $k(t)$ satisfies the condition $(\mathrm{K})$. Then the strong solution $u(t)$ can be represented in the form

$$
u(t)=S(t) k(0)+\int_{0}^{t} S(t-\tau) \dot{k}(\tau) d \tau
$$

8. The resolvent $R_{\delta}$. The representation (6.2) requires the derivative of $k(t)$ to exist and to satisfy certain other conditions. In this section we present another representation for the solution, one for which nothing is assumed about $\dot{k}(t)$ but which requires, instead, that $A^{1+\varepsilon} k(t)$ be integrable for some $\varepsilon>0$. The kernel of this representation is defined by

$$
R_{\delta}(t)=A^{1-\delta}\left[h(0) S(t)+\int_{0}^{t} h(t-\tau) S(\tau) d \tau\right] \quad(0<\delta \leqq 1)
$$


and will be called the $\delta$-resolvent of (6.1). By Theorem 6,

$$
R_{\delta} \in L^{p}(0, T ; B(X)) \text { if } p(1-\delta)<1 .
$$

Definition. We say that $k(t)$ belongs to the class $\AA_{\delta}^{\prime \prime}$ if $k(t) \in C^{0}([0, \infty) ; X)$, $k(t) \in D\left(A^{\delta}\right), A^{\delta} k(t) \in L^{q}(0, T ; X)$ for every $T>0$ and some $q>1 / \delta>0, e^{-\alpha t} A^{\delta} k(t)$ $\in L^{1}(0, \infty ; X)$ for some $\alpha>0$, and, finally, $A^{1+\varepsilon} k(t) \in L^{1}(0, T ; X)$ for every $T>0$ and some $\varepsilon>0$.

THeOREM 12. Let $h \in \mathfrak{S}_{1}, A \in \mathfrak{A}$ and let (2.1) hold. Then for any $k(t)$ in $\mathfrak{\Re}_{\delta}^{\prime \prime}$ the function

$$
u(t)=k(t)-\int_{0}^{t} R_{\delta}(t-\tau) A^{\delta} k(\tau) d \tau
$$

is a solution of (6.1), i.e., $u$ and $A u$ belong to $L^{1}(0, T ; X)$ for every $T>0$ and (6.1) holds for all $t>0$.

Proof. By Theorem 6, the Laplace transform $R_{\delta}(s)$ of $R_{\delta}(t)$ exists for all $s$ with $\operatorname{Re} s>0$. Applying $A^{\delta-1}$ to both sides of (8.1) and taking Laplace transforms, we get (since $A^{\delta-1}$ is bounded)

$$
A^{\delta-1} R_{\delta}(s)=\left[h(0)+h^{\wedge}(s)\right] S^{\wedge}(s)=h^{\wedge}(s)\left[I+h^{\wedge}(s) A\right]^{-1}
$$

where, in the last equality, we have used (2.18). Thus,

$$
R_{\delta}(s)=h^{\wedge}(s) A^{1-\delta}\left[I+h^{\wedge}(s) A\right]^{-1} \text {. }
$$

Observe next that, by (6.7), $A^{\delta} R_{\delta} k=R_{\delta} A^{\delta} k$. Using the fact that $A^{\delta}$ is closed, we can write (8.2) in the form

$$
u(t)=k(t)-A^{\delta} \int_{0}^{t} R_{\delta}(t-\tau) k(\tau) d \tau .
$$

Applying $A^{-\delta}$ to both sides and taking Laplace transforms, we obtain, after applying $A^{\delta}$ to the resulting equation,

$$
u^{\wedge}(s)=\left[I-A^{\delta} R_{\delta}^{\wedge}(s)\right] k^{\wedge}(s), \text { if } \operatorname{Re} s>\alpha .
$$

Substituting $R_{\delta}$ from (8.3), we get

$$
\widehat{u^{\wedge}}(s)=\left\{I-h^{\wedge}(s) A\left[I+h^{\wedge}(s) A\right]^{-1}\right\} k^{\wedge}(s)=\left[I+h^{\wedge}(s) A\right]^{-1} k^{\wedge}(s),
$$

i.e., $\left[I+h^{\wedge}(s) A\right] u^{\wedge}(s)=k^{\wedge}(s)$.

By the Corollary to Lemma 1 we then have

$$
u(t)+A \int_{0}^{t} h(t-\tau) u(\tau) d \tau=k(t)
$$

Using now the fact that $A^{1+\varepsilon} k(t) \in L^{1}(0, T ; X)$ for some $\varepsilon>0$ and any $T>0$, we can proceed as in the derivation of (6.7) and the argument following it and show 
that $A u(t) \in L^{1}(0, T ; X)$ for any $T>0$. Since $A$ is closed, (6.1) then follows from (8.4).

REMARK. If one removes the condition that $e^{-\alpha t} A^{\delta} k(t) \in L^{1}(0, \infty ; X)$ for some $\alpha>0$, then the right-hand side of (8.2) is still a solution of (6.1). Furthermore, this solution coincides with both the local $L^{\infty}$-solution and the strong solution if $h \in \mathfrak{S}^{\prime}, k \in \mathfrak{\Re}_{\mu}^{\prime}$.

The proofs of these statements are similar to the proofs of Corollary 2 to Theorem 9 and of Theorem 11.

9. Asymptotic behavior. The integral representations (7.6), (8.2) together with the bounds that have been established before for $S(t)$, can be used to obtain results on the asymptotic behavior of the solutions of (6.1). These results are analogous to theorems obtained by Friedman [3], [5] in the case where $A(t)$ is scalar.

Definition. Let $v(t)$ be a function from $[0, \infty)$ into $X$ and let $\phi(t)$ be a periodic function from $(-\infty, \infty)$ into $X$, i.e., $\phi(t+\sigma) \equiv \phi(t)$ for some $\sigma>0$. We say that $v$ is asymptotically periodic with limit $\phi$ if $\|v(t)-\phi(t)\| \rightarrow 0$ as $t \rightarrow \infty$.

TheOREM 13. Let $h \in \mathfrak{S}_{1}, A \in \mathfrak{A}, k \in \mathfrak{\Re}_{\mu}$ and let (2.1) hold. If there exists a bounded periodic function $\psi(t)$ from $(-\infty, \infty)$ into $X$, locally integrable and such that

$$
\int_{0}^{\infty}\|\dot{k}(t)-\psi(t)\| d t<\infty
$$

then the local $L^{\infty}$-solution of (6.1) is asymptotically periodic with the limit

$$
\psi_{0}(t) \equiv-\int_{0}^{\infty} S(\tau) \psi(t-\tau) d \tau
$$

Proof. It is obvious that $\psi_{0}(t)$ is periodic. By (6.2),

$$
u(t)=S(t) k(0)+\int_{0}^{t} S(t-\tau)[\dot{k}(\tau)-\psi(\tau)] d \tau+\int_{t}^{\infty} S(\tau) \psi(t-\tau) d \tau+\psi_{0}(t) .
$$

By Theorems 5, 2, the first and third terms on the right-hand side converge to zero if $t \rightarrow \infty$. It remains to prove that also the second term, call it $J(t)$, converges to zero if $t \rightarrow \infty$. Writing

$$
J(t) \equiv \int_{0}^{t}=\int_{0}^{N}+\int_{N}^{t}
$$

and using (9.1) and the fact that $\|S(t)\| \rightarrow 0$, we find that, for any $\varepsilon>0$,

$$
\|J(t)\|<\varepsilon \text { if } N=N(\varepsilon)
$$

is suitably chosen and $t$ is sufficiently large. Hence, $\|J(t)\| \rightarrow 0$ if $t \rightarrow \infty$.

In the special case where $\psi(t) \equiv 0$, we have:

Let $h \in \mathfrak{S}_{1}, A \in \mathfrak{A}, k \in \mathfrak{I}_{p, \mu}$ for some $p<\infty$, and let (2.1) hold. Then the $L^{q}$-solution of (6.2) satisfies: $\|u(t)\| \rightarrow 0$ if $t \rightarrow \infty$.

Indeed, this is a part of Theorem 9. 
TheOREM 14. Let $h \in \mathfrak{S}_{1}, A \in \mathfrak{A}$ and let (2.1) hold. Let $k(t) \in \mathfrak{\Omega}_{1}^{\prime \prime}$ and let $k(t)$ be asymptotically periodic with limit $\phi(t)$, such that $A \phi(t)$ is bounded, and locally integrable and

$$
\int_{0}^{\infty}\|A k(t)-A \phi(t)\| d t<\infty .
$$

Then the solution (8.2) of (6.1) is asymptotically periodic with the limit

$$
\phi_{0}(t) \equiv-\int_{0}^{\infty} R_{1}(\tau) A \phi(t-\tau) d \tau
$$

The proof is similar to the proof of Theorem 13 provided we show that $\left\|R_{1}(t)\right\| \rightarrow 0$ as $t \rightarrow \infty$. But this follows from (8.1), since $h \in L^{1}(0, \infty)$ and $\|S(t)\| \rightarrow 0$ as $t \rightarrow \infty$.

Similar results can be stated with $R_{\delta}, \delta>0$.

Note that the condition $e^{-\alpha t} A^{\delta} k(t) \in L^{1}(0, \infty ; X)$ is superfluous (by the remark at the end of $\S 8$ ).

Generalizations. Most of the results of Chapter 2 can be generalized to include integral equations of the form

$$
u(t)=k(t)-\int_{0}^{t} A(t-\tau) u(\tau) d \tau
$$

Here, $A(t)$ is a bounded operator on $X$ that is strongly differentiable and such that $A(t) \in L^{1}(0, \infty ; B(X))$. Of course, appropriate restrictions on the spectrum of $A(t)$ must also be made.

These generalizations allow one to study the case of systems of scalar equations which arise when $A(t)$ is a matrix of functions.

\section{REFERENCES}

1. S. Agmon, On the eigenfunctions and on the eigenvalues of general elliptic boundary value problems, Comm. Pure Appl. Math. 15 (1962), 119-147.

2. N. Dunford and J. Schwartz, Linear operators, Part I, Interscience, New York, 1964.

3. A. Friedman, On integral equations of Volterra type, J. Analyse Math. 11 (1963), 381-413.

4. - Uniqueness of solutions of ordinary differential inequalities in Hilbert space, Arch. Rational Mech. Anal. 17 (1964), 353-357.

5. - Periodic behavior of solutions of Volterra integral equations, J. Analyse Math. 15 (1965), 287-303.

6. - Differentiability of solutions of ordinary differential equations in Hilbert space, Pacific J. Math. 16 (1966), 267-271.

7. V. P. Glushko and S. G. Krein, Fractional power of differential operators and inclusion theorems, Dokl. Akad. Nauk SSSR 122 (1958), 963-966.

8. E. Hille and R. S. Phillips, Functional analysis and semi-groups, Amer. Math. Soc. Colloq. Publ. Vol. 31, Amer. Math. Soc., Providence, R. I., 1957.

9. T. Kato, Integration of the equation of evolution in a Banach space, J. Math. Soc. Japan 5 (1953), 208-234.

10. - On linear differential equations in Banach spaces, Comm. Pure Appl. Math. 9 (1956), 479-486. 
11. —-, Fractional powers of dissipative operators, J. Math. Soc. Japan 13 (1961), 246-274.

12. H. Komatzu, Abstract analyticity in time and unique continuation property for solutions of parabolic equations, J. Fac. Sci. Univ. Tokyo Sect. I 9 (1961), 1-11.

13. R. S. Phillips, Perturbation theory for semi-groups of linear operators, Trans. Amer. Math. Soc. 74 (1954), 199-221.

14. M. Shinbrot and S. Kaniel, The initial value problem for the Navier-Stokes equations, Arch. Rational Mech. Anal. 21 (1966), 270-285.

15. P. E. Sobolevskir, On equations of parabolic type in a Banach space, Trudy Moscov. Mat. Obšc. 10 (1961), 297-350=Amer. Math. Soc. Transl. (2) 49 (1965), 1-62.

16. H. Tanabe, $A$ class of the equations of evolution in a Banach space, Osaka Math. J. 11 (1959), 121-145.

17. - Remarks on the equations of evolution in a Banach space, Osaka Math. J. 12 (1960), 145-166.

18. - On the equations of evolution in a Banach space, Osaka Math. J. 12 (1960), 363-376.

19. E. C. Titchmarsh, Fourier integrals, Oxford Univ. Press, Oxford, 1950.

20. K. Yosida, Fractional powers of infinitesimal generators and the analyticity of semi-groups generated by them, Proc. Japan Acad. 36 (1960), 86-89.

NORTHWESTERN UNIVERSITY,

Evanston, ILlinoIs 